\title{
Residual vibration reduction in back-and-forth moving systems driven by slider-crank mechanisms working through a dead point configuration
}

\author{
Joaquim M. Veciana Fontanet ${ }^{1 *}$, Lluïsa Jordi Nebot ${ }^{1}$, Eduard Lores García ${ }^{1}$ \\ 1 Department of Mechanical Engineering, Universitat Politècnica de Catalunya, \\ Diagonal 647, Barcelona 08028, Spain. \\ * Corresponding author.
}

E-mail addresses: joaquim.maria.veciana@upc.edu (Joaquim M. Veciana Fontanet), lluisa.jordi@upc.edu (Lluïsa Jordi Nebot), eduard.lores@.upc.edu (Eduard Lores García).

\begin{abstract}
This study proposes an algorithm to construct back-and-forth motion profiles to reduce residual vibrations of 1-dof linear oscillatory systems, driven through a transmission chain made up of a 1-dof linkage mechanism. This set can be advantageous for high transmission ratios, when multistage drive trains are discouraged to prevent a build-up of backlash nonlinearities. We assume that the mechanism: $i$ ) is moved by a conventional electric actuator and, $i i$ ) is working through a dead point configuration. The solution focuses on the continuity degree $C^{n}$ of the motion profile to guarantee its feasibility by means of the mentioned actuators. We aim to provide an algorithm to design these profiles. The development meshes several conclusions from other studies: the classical strategies of residual vibration reduction together with an analytical solution, with regard to $C^{n}$, of the inverse kinematic problem at a dead point configuration. The development includes an analytical approach to some methods of residual vibration reduction, in addition to numerical simulations. Finally, we present experimental results on a slider-crank mechanism test bed, with a pendulum acting as the oscillatory system.
\end{abstract}

\section{Keywords}

Residual vibration, motion profile, motion law, slider-crank mechanism, dead point.

\section{Introduction}

The rise of robotics and automation makes the design of machine element motion profiles an interesting area of research. In particular, the effectiveness of residual vibration reduction has been widely studied [1], [2], [3]. Due to transient excitations, the oscillatory behavior of a system may be noticeable and thus, its normal operation may be compromised even beyond the end of the transient excitation. Within these studies, extensive work has been carried out on discrete linear systems among the so-called feed-forward methods, based mainly on time-domain and frequency-domain approaches.

According to the coordinates indicated in Figure 1, it is common that the transmission chain present in most mechanical systems has a constant ratio $\tau=q^{\mathrm{i}} / q^{\mathrm{d}}$ (Figure 1,a), such as gear trains - or near constant, such as chains or pulley-belt systems. In this context, $q_{\mathrm{c}}^{\mathrm{d}}(t), q^{\mathrm{d}}(t), q^{\mathrm{i}}(t)$ and $y(t)$ are the input command referring to the dependent coordinate, the dependent coordinate itself, the independent coordinate and the system response, respectively. Therefore, if $q_{\mathrm{c}}^{\mathrm{d}}(t)$ fulfills the general rules of dynamics, the transmission chain output $q^{\mathrm{i}}(t)$ is quite similar to the input command, with the limitations that come from the filtering effect of the system. However, if the user wants 
to avoid residual vibrations of the response $y(t)$ of the oscillatory system, the backlash added to the low stiffness of the aforementioned transmissions can be a major drawback; they may provoke unwanted excitation of vibratory modes, especially with high transmission ratios having more than one stage. In these cases, it could be advantageous to use a linkage mechanism around the dead point configuration, to achieve the desired ratio without excessive play. Figure 1,b) shows a slider-crank mechanism in a generic configuration that, within this scenario, can be used instead of the mechanism $a$ ). Assuming a system with a linkage mechanism working through a dead point of coordinate $q^{\mathrm{i}}$, the purpose of this paper is to define the necessary conditions for a time-domain motion profile $q^{\mathrm{i}}(t)$ to: $i$ ) be able to reduce residual vibrations at the output $y(t)$ of a discrete linear oscillatory system, and ii) be feasible with a conventional electric actuator commanded by $q_{\mathrm{c}}^{\mathrm{d}}(t)$.

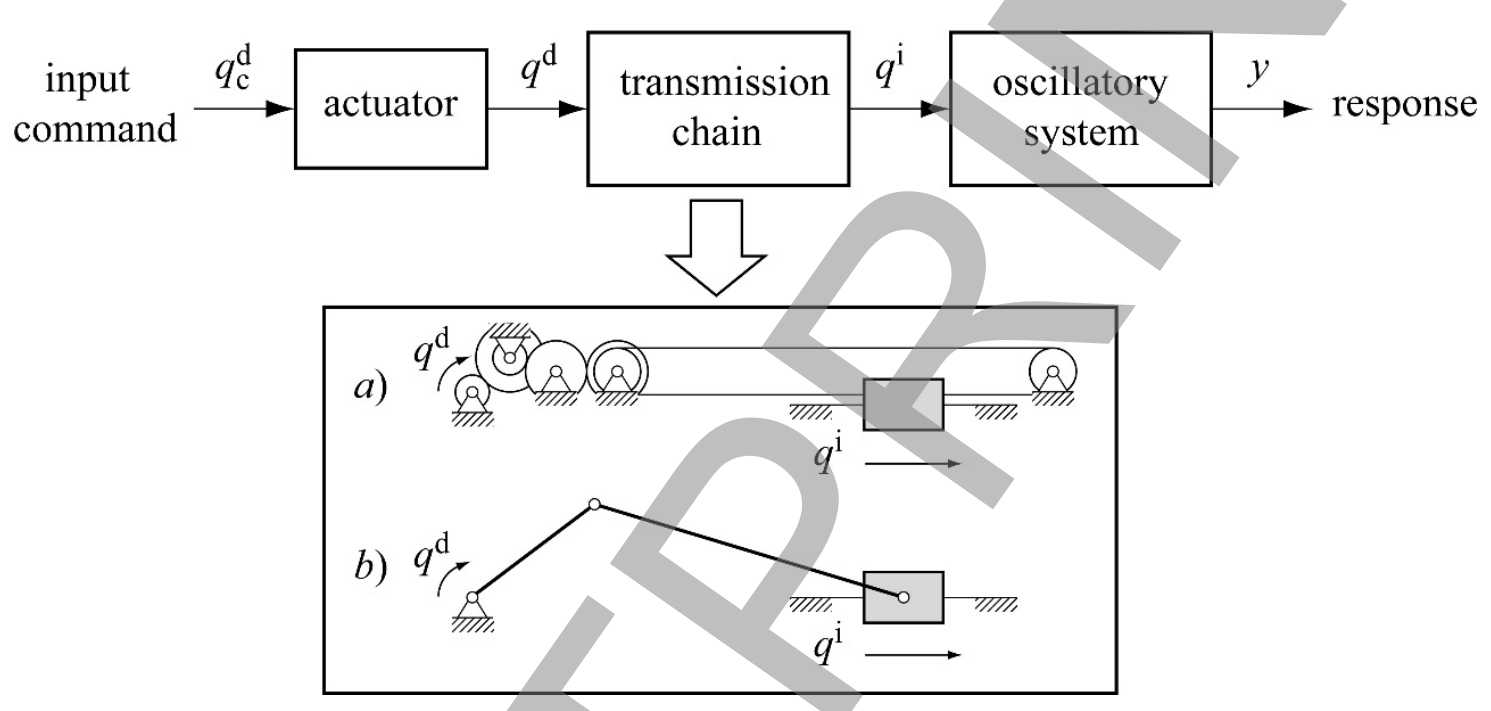

Figure 1. Block diagram of a system with transmission chain, a) linear: gears and belt, and $b$ ) non-linear: linkage mechanism.

The most successful time-domain techniques for reducing residual vibrations started during the 1950s. Smith proposed the posicast control [4], being the basis for the Zero Vibration (ZV) input shapers. They are so-called because it has been proven analytically that the residual vibration is null after the transient input. In this technique, an input command is formed by a sequence of two impulses, properly located in time and with the appropriate amplitude. The superposition of the two oscillatory system responses results in a non-oscillating signal after the second impulse. To generate feasible inputs, we need to convolve an arbitrary command (the unshaped command) with this sequence in the time domain. In this way, Smith proved that the vibration cancellation properties remain invariable in the resultant signal.

The effectiveness of $\mathrm{ZV}$ is very sensitive to the correct estimation of the natural frequency and damping ratio of the oscillatory system. By adding a new impulse to the sequence, Singer and Seering [5] developed Zero Vibration and Derivative (ZVD) input shapers, which provide built-in robustness to the command with regard to the variation of system parameters. By adding more impulses, they further increase the robustness (ZVDD, ZVDDD...). Nevertheless, the duration of the input command increases, as well. To provide robustness with shorter commands, Singhose et al. [7] propose the Extra Insensitive (EI) technique, which through phasorial analysis, is able to define the percentage of residual vibration allowed by the user with only a three-impulse sequence. A similar method - the Specified Insensitive Shaper (SI) - is presented by Singer and 
Seering [9] and Singhose et al. [10] where, in addition to fixing the percentage of residual vibrations, the user also defines the desired robustness. Singhose et al. [8] also add negative impulses to decrease the duration of the impulse sequences. Hyde and Seering [6] extend the method to cancel multiple-mode oscillations by obtaining an impulse sequence as a result of the time-domain convolution of several sequences, each designed for its individual mode.

With regard to methods that use the frequency domain to generate input commands, Meckl and Seering [11], [12] prove that the amplitude of the residual vibration is proportional to the spectral magnitude of the input command (at the natural frequency of the system) only for undamped systems. They study ramped sinusoidal signals avoiding magnitude contribution around the system's natural frequency. Meckl et al. [13] develop s-curves with a ramp-up shape to use as velocity profiles by analyzing their frequency content to minimize the response time and residual vibration. Bhat and Miu [15] and Bhat et al. [16] demonstrate that the necessary and sufficient condition that an input command should accomplish to obtain zero residual vibration is that the Laplace Transform of the transient command be null at the oscillatory system poles. Taking advantage of this control strategy, called zero-placement, Veciana and Cardona [18] developed a zero vibration method for damped systems. The input command is designed to have (in the frequency domain) a null magnitude at the system's natural frequency; later it is multiplied by a negative exponential time function, which includes the damping ratio. Tuttle and Seering [17] and Murphy and Watanabe [19] both extend the zero-placement technique to the discrete domain and for multiple-mode systems.

Among frequency-domain methods, conventional filtering is also studied to reduce residual vibrations. Singhose et al. [14] compare ZV techniques with IIR low-pass filters (Chebyshev, Butterworth, and elliptic), FIR low-pass filters (Parks-McClellan, Hamming), and notch filters. Singhose and Vaughan [20] compare EI and SI shapers with FIR filters as well. As mentioned above, the proportionality between the residual vibration and the frequency content of the input command is only valid for undamped systems. Therefore, for damped ones these methods are not analytically proven to be zero-vibration and are resultantly less effective.

The present study proposes an algorithm to construct motion profiles to reduce residual vibrations for a one degree-of-freedom oscillatory system, when the transmission chain is a linkage mechanism moved by a conventional electric actuator. The mechanisms used in this study are those whose elements are linked with lower pairs and do not present redundancies. The algorithm focuses on the continuity degree $C^{n}$ of the motion profile [21], which is a key parameter to guarantee that it can be reproduced by the aforementioned actuators. The novelty of this study is the algorithm provided to the user to facilitate the design of these profiles. It assumes that mechanisms are working through a dead point to take advantage of the high transmission ratio for this configuration. This study meshes several conclusions from other studies to come up with a solution to the problem stated. It uses the classical strategies of residual vibration reduction, together with an analytical solution-with regard to $C^{n}$ - of the inverse kinematic problem at a dead point configuration. The main contributions of this development are: $i$ ) the specific rules and the algorithm to design the aforementioned motion profiles, taking into consideration the limitations of actuators and the working configuration, $i$ ) the analysis of the effect of the convolution operation between an arbitrary function and a sequence of impulses or a pulse, on the continuity of the resultant function, and iii) the validation of the conclusions obtained through some simulation examples and experimental results. 
The paper is organized as follows: Section 2 describes the basic preliminaries used in its development with summarized rules and conclusions for each study. Section 3 introduces the problem formulation to obtain motion profiles that reduce residual vibrations when the transmission chain is a linkage mechanism working through a dead point configuration. It focuses on two methods referred to in the literature and presents an algorithm and some simulation examples. Section 4 shows an experimental test-bed and several results that validate the usefulness of our development. Finally, we draw conclusions in Section 5.

\section{Preliminaries}

The formulation of this study has been developed by means of the example shown in Figure 2 and the nomenclature according to Figure 1. A second order harmonic oscillator is controlled through its base with a slider-crank mechanism working through a dead point configuration. Nevertheless, the conclusions obtained are valid for any one degree-of-freedom linkage mechanism driving any arbitrary one degree-of-freedom oscillatory system.

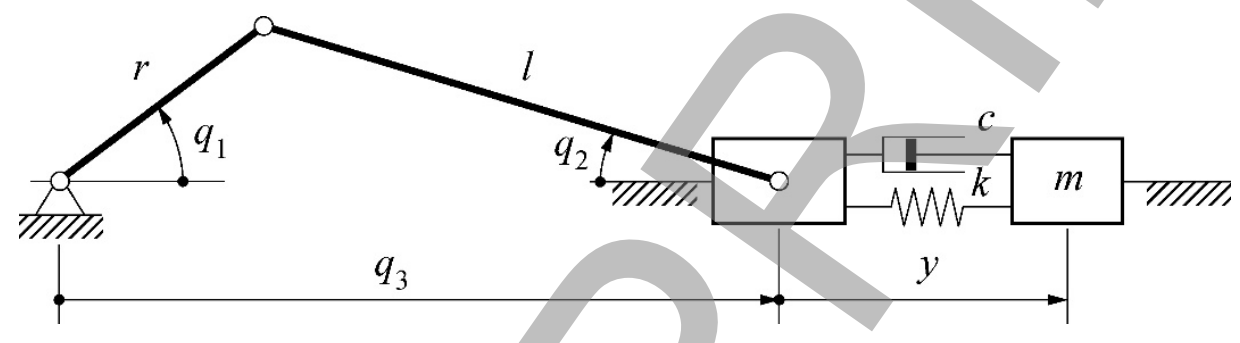

Figure 2. Linear oscillatory system controlled through a slider-crank mechanism.

\subsection{Capability of conventional actuators}

In order to assess the capability of conventional electric actuators to reproduce an input command with reasonable precision, we analyze the minimum requirements of this signal with regard to the continuity degree $C^{n}$ in previous works [22], [23]. In this way we study the limitations caused by the discontinuities in position, velocity, and acceleration. The study does not carry out a quantitative analysis, i.e., the frequency-response range of the actuator is not considered; that will depend mainly on the type of motor used.

The overall electrical time constant of an actuator can be split into the plain electrical time constant and magnetic diffusion time, being about a few milliseconds for conventional electric actuators (AC and DC servomotors) [24]. Therefore, if we assume a voltage step input, a steady stationary torque, then consequently, a steady stationary acceleration can be achieved after a transition time $t_{\mathrm{t}}$ in practice.

According to this statement, we assume a motion profile $q_{\mathrm{c}}^{\mathrm{d}}(t)$, which is described by Equation (1):

$q_{\mathrm{c}}^{\mathrm{d}}(t)=\left\{\begin{array}{cc}0 & -\infty<t \leq t_{0^{-}} \\ a t+b t^{2} & t_{0^{+}} \leq t<+\infty\end{array} ; \dot{q}_{\mathrm{c}}^{\mathrm{d}}(t)=\left\{\begin{array}{cc}0 & -\infty<t \leq t_{0^{-}} \\ a+2 b & t_{0^{+}} \leq t<+\infty\end{array} ; \ddot{q}_{\mathrm{c}}^{\mathrm{d}}(t)=\left\{\begin{array}{cc}0 & -\infty<t<t \\ a \delta(t) & t_{0^{-}} \leq t \leq t_{0^{+}} \\ 2 b & t_{0^{+}}<t<+\infty\end{array}\right.\right.\right.$

where $\delta(t)$ is the Dirac delta function, $t_{0-}$ and $t_{0+}$ are, respectively, the time instants just before and just after the motion starts, $t_{0}$, and $a$ and $b$ are constant coefficients of the generic polynomial that describes $q_{\mathrm{c}}^{\mathrm{d}}(t)$. If $a=0$ and $b \neq 0$, the motion profile has a 
continuity degree of $C^{1}$, which implies an acceleration step, and hence it can be reproduced with reasonable precision after a transition time $t$ t. This transition time is related to the above-mentioned overall electrical time constant and hence, to the frequency range where the actuator is able to respond. Figure 3,a), b) and c) show, $q_{\mathrm{c}}^{\mathrm{d}}(t)$, and its time derivatives, respectively (blue solid lines). Figure $3, c$ ) also includes the real motion (dashed line) and the transition time $t_{\mathrm{t}}$ is remarked.

Nonetheless, if the motion profile $q_{\mathrm{c}}^{\mathrm{d}}(t)$ is described with a function that has a continuity degree of $C^{0}$, the acceleration contains an impulse. Although this kind of time-domain profile can be physically approximated by a percussive torque, the majority of electric actuators are not able to reproduce it. In this case, $q_{c}^{d}(t)$ and its time derivatives follow red lines in Figure 3, and are described by Equation (1) for $a \neq 0$ and $b=0$.

At this point, the first statement can be established:

Statement 1: in the context of this study, we consider acceptable the hypothesis that a conventional electric actuator is able to reproduce input commands $q_{\mathrm{c}}^{\mathrm{d}}(t)$ with a minimum continuity degree of $C^{1}$.

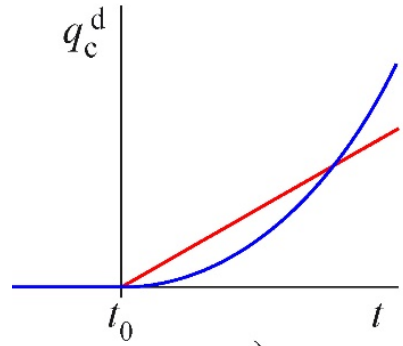

a)

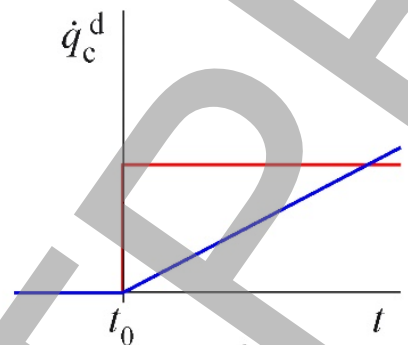

b)

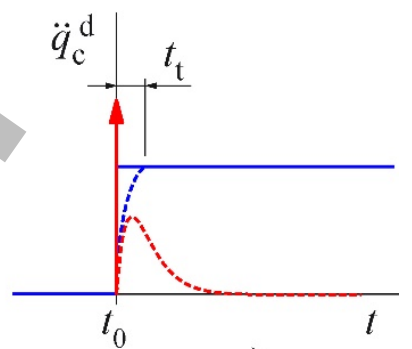

c)

Figure 3. Input commands $(a)$ and time derivatives ( $b$ and $c$ ), with a discontinuity in acceleration (blue line) and in velocity (red line), and their real motions (dashed lines), respectively.

\subsection{Feasibility of motion laws for planar one degree-of-freedom linkage mechanisms at dead point configurations}

In linkage mechanisms, it is common to describe their configuration with a non-minimum set of $r+m$ coordinates $q_{i}$, where $i=1 \ldots, r+m$, which are the generalized coordinates. The minimum number of coordinates required to describe the configuration is the number $m$ of independent coordinates $q^{\mathrm{i}}$, and the remaining $r$ are the dependent coordinates $q^{\mathrm{d}}$. Therefore, a set of $r$ constrained equations $\boldsymbol{\Phi}(q)=0$ - usually nonlinear - can be formulated among them. The evolution of these coordinates usually presents singular configurations, depending on the geometric characteristics and dimensions of the mechanism. One type of these unique configurations is the so-called dead point of a coordinate, in which it reaches its maximum or minimum value [25], [26].

A one degree-of-freedom mechanism has a unique $(m=1)$ independent coordinate $q^{\mathrm{i}}$. For such mechanisms, the time-domain evolution of this coordinate $q^{\mathrm{i}}(t)$ is usually set by the user to fulfill a determined output or motion profile. Nevertheless, it is common that the actuators control the system through another coordinate $q^{\mathrm{d}}$, other than the independent one. Consequently, it is necessary to determine the temporal evolution of $q^{\mathrm{d}}(t)$ from the desired motion profile $q^{\mathrm{i}}(t)$ by means of the system constraint equations 
(Equation (2)), i.e., the inverse kinematic of the mechanism must be solved. According to [22], [23], by differentiating Equation (2), the dependent velocities and accelerations can be calculated following Equations (3) and (4), respectively.

$$
\begin{gathered}
\boldsymbol{\Phi}(\boldsymbol{q})=\left\{\boldsymbol{\Phi}_{1}(\boldsymbol{q}) \ldots \boldsymbol{\Phi}_{r}(\boldsymbol{q})\right\}^{\mathrm{T}}=0 \\
\dot{\boldsymbol{q}}^{\mathrm{d}}=-\frac{\operatorname{adj}\left(\boldsymbol{\Phi}_{\mathrm{q}}^{\mathrm{d}}\right)}{\operatorname{det}\left(\boldsymbol{\Phi}_{\mathrm{q}}^{\mathrm{d}}\right)} \boldsymbol{\Phi}_{\mathrm{q}}^{\mathrm{i}} \dot{q}^{\mathrm{i}} \\
\ddot{\boldsymbol{q}}^{\mathrm{d}}=-\frac{\operatorname{adj}\left(\boldsymbol{\Phi}_{\mathrm{q}}^{\mathrm{d}}\right)}{\operatorname{det}\left(\boldsymbol{\Phi}_{\mathrm{q}}^{\mathrm{d}}\right)}\left[\boldsymbol{\Phi}_{\mathrm{q}}^{\mathrm{i}} \ddot{q}^{\mathrm{i}}+\dot{\boldsymbol{\Phi}}_{\mathrm{q}} \dot{\boldsymbol{q}}\right]
\end{gathered}
$$

where $\boldsymbol{\Phi}_{\mathrm{q}}^{\mathrm{d}}$ is de jacobian matrix of the dependent coordinates, $\boldsymbol{\Phi}_{\mathrm{q}}^{\mathrm{i}}$ is de jacobian matrix of the independent coordinates and $\dot{\boldsymbol{\Phi}}_{\mathrm{q}}$ is the temporal derivative of the jacobian matrix. As mentioned in Section 1, in common transmission chains such as gear trains, chains or pulley-belt systems, the ratio $q^{\mathrm{i}}(t) / q^{\mathrm{d}}(t)$ is practically constant and hence, linear. However, when the transmission chain is composed of a linkage mechanism, this relation is not linear and has singularities, such as the dead points of the output coordinate $q^{\mathrm{i}}(t)$. In previous works [22], [23], the authors analyzed the continuity condition that must accomplish a generic motion profile when the mechanism is controlled by a conventional electric actuator, with the limitations specified in Statement 1. These studies focused on the motion profile described by $q^{\mathrm{i}}(t)$, moving through a dead point, to be feasible by $q^{\mathrm{d}}(t)$, which is the coordinate driven by the actuator.

\begin{tabular}{ccc} 
& Case 1 & Case 2 \\
\hline $\begin{array}{c}\text { Requirements } \\
\text { through a dead point }\end{array}$ & $\dot{q}^{\mathrm{i}}\left(t_{\mathrm{dp}}\right)=0$ & $\dot{q}^{\mathrm{i}}\left(t_{\mathrm{dp}}\right)=0$ \\
& $\ddot{q}^{\mathrm{i}}\left(t_{\mathrm{dp}}\right) \neq 0$ & $\ddot{q}^{\mathrm{i}}\left(t_{\mathrm{dp}}\right)=0$ \\
\hline $\begin{array}{c}\text { Minimum continuity } \\
\text { of a motion profile }\end{array}$ & $\dddot{q}^{\mathrm{i}}\left(t_{\mathrm{dp}}\right)=0$ \\
\hline
\end{tabular}

Table 1. Two cases of motion profile continuity requirements of the independent coordinate $q^{\mathrm{i}}(t)$, to be feasible through a dead point configuration, when $q^{\mathrm{d}}(t)$ is controlled by a conventional electric actuator.

Statement 2: the results reached by the authors are shown in Table 1, where $t_{\mathrm{dp}}$ means the instant when the corresponding coordinate reaches the dead point. The continuity degree and the requirements at the dead point must follow either of the indicated cases to construct a feasible motion profile $q^{\mathrm{i}}(t)$.

In the example of the mechanism illustrated in Figure 2, the independent coordinate $q^{\mathrm{i}}$ is assumed to be $q_{3}$, which is the position coordinate from the crank revolute joint to the slider. The dependent coordinates $\boldsymbol{q}^{\mathrm{d}}$ are $\left\{q_{1} q_{2}\right\}^{\mathrm{T}}$, which correspond to the angular coordinates of the crank and the coupler, respectively. In this case, Equations (2), (3), and (4) yield, correspondingly: 


$$
\begin{aligned}
& \boldsymbol{\Phi}\left(q_{1}, q_{2}, q_{3}\right)=\left\{\begin{array}{l}
\Phi_{1}\left(q_{1}, q_{2}, q_{3}\right) \\
\Phi_{2}\left(q_{1}, q_{2}, q_{3}\right)
\end{array}\right\}=\left\{\begin{array}{l}
r \sin q_{1}-l \sin q_{2} \\
-r \cos q_{1}-l \cos q_{2}+q_{3}
\end{array}\right\}=0 \\
& \left\{\begin{array}{l}
\dot{q}_{1} \\
\dot{q}_{2}
\end{array}\right\}=-\frac{1}{r l \sin \left(q_{1}+q_{2}\right)}\left[\begin{array}{cc}
l \sin q_{2} & l \cos q_{2} \\
-r \sin q_{1} & r \cos q_{1}
\end{array}\right]\left\{\begin{array}{l}
0 \\
1
\end{array}\right\} \dot{q}_{3} \\
& \left\{\begin{array}{l}
\ddot{q}_{1} \\
\ddot{q}_{2}
\end{array}\right\}=-\frac{1}{r l \sin \left(q_{1}+q_{2}\right)}\left[\begin{array}{cc}
l \sin q_{2} & l \cos q_{2} \\
-r \sin q_{1} & r \cos q_{1}
\end{array}\right]\left[\begin{array}{l}
0 \\
1
\end{array}\right\} \ddot{q}_{3}+ \\
& \left.\left[\begin{array}{ccc}
-\dot{q}_{1} r \sin q_{1} & \dot{q}_{2} l \sin q_{2} & 0 \\
\dot{q}_{1} r \cos q_{1} & \dot{q}_{2} l \cos q_{2} & 0
\end{array}\right]\left\{\begin{array}{l}
\dot{q}_{1} \\
\dot{q}_{2} \\
\dot{q}_{3}
\end{array}\right\}\right]
\end{aligned}
$$

\section{Definition of motion displacement profiles through a dead point configuration to reduce residual vibrations}

With regard to Figure 1, motion profiles defined for the coordinate $q^{\mathrm{i}}(t)$ must fulfill some characteristics to reduce residual vibrations of the output of an oscillatory system, $y(t)$, when $q^{\mathrm{i}}(t)$ is working through a dead point configuration.

Take for example, Figure 2. Following the outlined coordinates, where the coordinate $q_{3}(t)$ is assumed to be $q^{\mathrm{i}}(t)$, and focusing just on the oscillatory system, the motion equation is:

$$
\ddot{y}+2 \zeta \omega_{0} y+\omega_{0}^{2} y=-\ddot{q}_{3}
$$

where $\omega_{0}=\sqrt{k / m}$ is the system's natural frequency and $\zeta=c / \sqrt{4 k m}$ is the damping ratio. The impulse response of the system, when the input $\dot{q}_{3}$ and the output $y$ are described by velocity and position magnitudes, respectively, is:

$$
h(t)=-\frac{1}{\cos \psi} \mathrm{e}^{-\zeta \omega_{0} t} \cos \left(\omega_{\mathrm{d}} t+\psi\right)
$$

with $\omega_{\mathrm{d}}=\omega_{0} \sqrt{1-\zeta^{2}}$ the system's oscillation frequency and $\psi=\arctan \left(\zeta / \sqrt{1-\zeta^{2}}\right)$.

Although most of the methods to reduce residual vibrations are compatible with the development carried out in this paper, we focus on a classic input shaper (Smith [4] and Singer and Seering [5]) and on a pulse time-convolution technique (Veciana and Cardona [18]).

In both methods, the resulting motion profile $q^{\mathrm{i}}(t)$ is obtained by a time-domain conyolution between the so-called unshaped command $u_{\mathrm{a}}(t)$ with another function $u \mathrm{~b}(t)$ that depends on the method used.

In the following examples, the unshaped command $u_{\mathrm{a}}(t)$ is defined through Bézier curves to easily set the desired continuity degree between sections by aligning some control points. According to Table $1, u_{\mathrm{a}}(t)$ must be at least $C^{2}$ in Case 1 , and $C^{3}$ in Case 2 , to obtain a $q^{\mathrm{i}}(t)$ that is feasible for conventional electric actuators. The degree $g$ and the $p$ control points of these curves have been adjusted accordingly. 


\subsection{Input shaping}

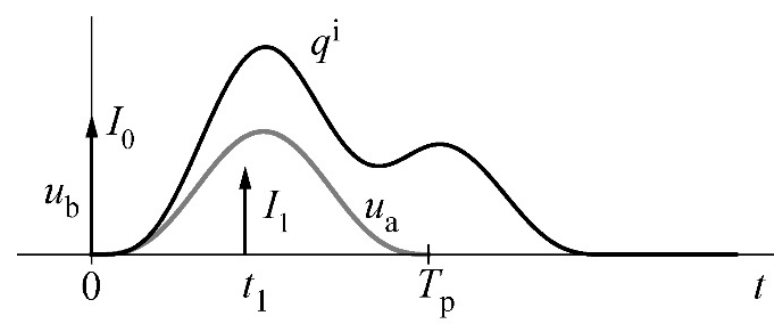

Figure 4. Two-impulse input shaping.

According to the input shaping method, a motion profile $q^{\mathrm{i}}(t)$ results from the time-convolution of an arbitrary unshaped command $u_{\mathrm{a}}(t)$ with a sequence of impulses $u$ b $(t)$ properly located in time (Figure 4$)$. The case of two-impulse input shaping yields:

$$
\begin{gathered}
u_{\mathrm{b}}(t)=I_{0} \delta(t)+I_{1} \delta\left(t-t_{1}\right) \quad t_{1}>0 \\
q^{\mathrm{i}}(t)=A\left(u_{\mathrm{a}}(t) \otimes u_{\mathrm{b}}(t)\right)+q^{\mathrm{i}}(0)
\end{gathered}
$$

in which $\otimes$ means convolution product, $\delta(t)$ is the Dirac delta function, $A$ is a constant value and

$$
\begin{gathered}
t_{1}=\frac{\pi}{\omega_{\mathrm{d}}} \\
\left(I_{0}, I_{1}\right)=\left(\frac{1}{1+K}, \frac{K}{1+K}\right) ; \quad K=\mathrm{e}^{\frac{-\zeta \pi}{\sqrt{1-\zeta^{2}}}}
\end{gathered}
$$

By using this technique, the continuity degree $C^{n}$ of the resulting function $q^{\mathrm{i}}(t)$ remains invariable with respect to the one from the unshaped command $u_{\mathrm{a}}(t)$. This statement is valid if $u_{\mathrm{a}}(t)$ is $C^{n}$ for all its domain, and $u_{\mathrm{a}}(t)$ and its $n$ first derivatives start and finish with null values (see Appendix).

To obtain a backward and forward displacement profile - the movement starts and finishes in the same position-it is necessary to know the initial coordinate $q^{\mathrm{i}}(0)$, the dead point coordinate $q^{\mathrm{i}}\left(t_{\mathrm{dp}}\right)$ and the oscillation system parameters $\omega_{0}$ and $\zeta$. The proposed algorithm is (Figure 5):

a) Select an unshaped profile $u_{\mathrm{a}}(t)$ that starts and finishes at a null value, with a minimum continuity $C^{2}$ (see Table 1 ) for all its definition range, including both ends.

b) Calculate $u_{\mathrm{b}}(t)$ with the oscillatory system parameters.

c) In order to guarantee the dead point configuration, calculate parameter $A$ through Equation (14) to scale the convolution product of Equation (11) suitably,

$$
A=\frac{q^{\mathrm{i}}\left(t_{\mathrm{dp}}\right)-q^{\mathrm{i}}(0)}{\max \left(u_{\mathrm{a}}(t) \otimes u_{\mathrm{b}}(t)\right)}
$$

d) Obtain $q^{\mathrm{i}}(t)$ using Equation (11) and $q^{\mathrm{d}}(t)$ with Equation (2).

e) Check if $\ddot{q}^{\mathrm{i}}\left(t_{\mathrm{dp}}\right) \neq 0$ (the majority of the times). Otherwise, we suggest to increase the minimum continuity of $u_{\mathrm{a}}(t)$ to $C^{3}$ (see Table 1 ) and repeat the sequence. In this case, if $\ddot{q}^{\mathrm{i}}\left(t_{\mathrm{dp}}\right)=0$, check if $\dddot{q}^{\mathrm{i}}\left(t_{\mathrm{dp}}\right)=0$. If not, the profile is not feasible. 


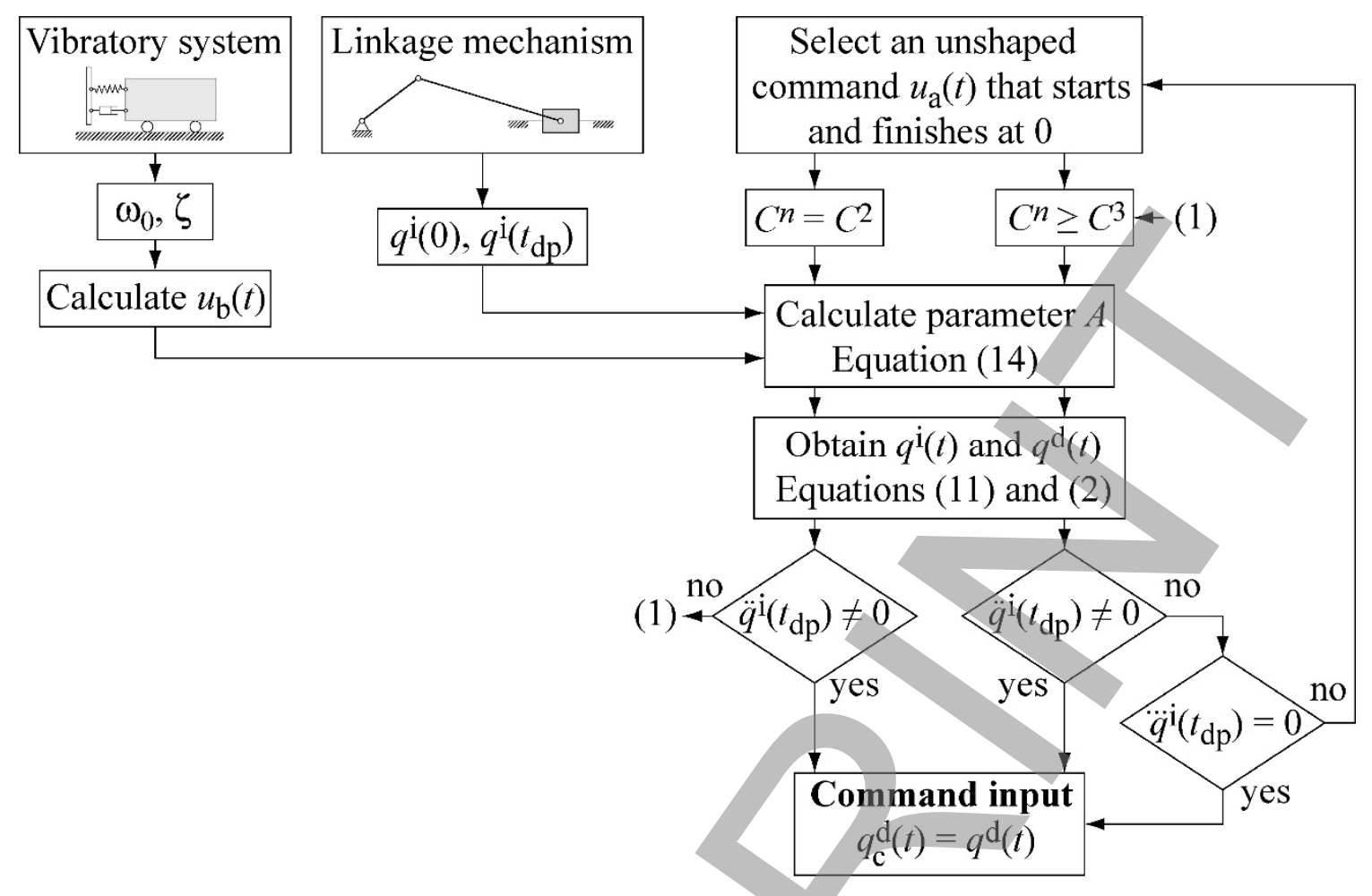

Figure 5. Algorithm flow diagram.

\section{Examples}

Input shaping, Case $1\left(\dot{q}^{\mathrm{i}}\left(t_{\mathrm{dp}}\right)=0\right.$ and $\left.\ddot{q}^{\mathrm{i}}\left(t_{\mathrm{dp}}\right) \neq 0\right)$ :

Figure 6 (red line) shows an example of the crank angular coordinate $q_{1}(t)$ that corresponds to a slider coordinate $q 3(t)$, which follows a $C^{2}$ displacement profile. This profile is constructed through a Bézier curve, $u_{\mathrm{a}}(t)$, with $g=6, p=7$ and a total duration $T_{\mathrm{p}}=1.0 \mathrm{~s}$ (see Equation (15)). An oscillatory system with $\omega_{\mathrm{d}}=2 \pi \mathrm{rad} / \mathrm{s}$ and $\zeta=0.1$ is assumed.

$$
u_{\mathrm{a}}(t)=\left\{\begin{array}{l}
0 \quad t<0 \\
\left(\frac{t}{T_{\mathrm{p}}}\right)^{3}\left(1-\frac{t}{T_{\mathrm{p}}}\right)^{3} \quad 0 \leq t<T_{\mathrm{p}} \\
0 \quad t \geq T_{\mathrm{p}}
\end{array}\right.
$$

Input shaping, Case $2\left(\dot{q}^{\mathrm{i}}\left(t_{\mathrm{dp}}\right)=0, \ddot{q}^{\mathrm{i}}\left(t_{\mathrm{dp}}\right)=0\right.$ and $\left.\dddot{q}^{\mathrm{i}}\left(t_{\mathrm{dp}}\right)=0\right)$ :

Figure 6 (blue lines) shows an analogous example to the former one, where $q_{3}(t)$ follows a displacement profile $C^{3}$ and $u_{\mathrm{a}}(t)$ is a symmetric piecewise profile made of Bézier curves $(g=7, p=8)$ and constant functions, as indicated by Equation (16) $\left(T_{\mathrm{p} 1}=0.383 \mathrm{~s}, T_{\mathrm{p} 2}=0.467 \mathrm{~s}\right.$ and $\left.T_{\mathrm{p}}=0.85 \mathrm{~s}\right)$. 


$$
\begin{aligned}
& \begin{cases}0 & t<0 \\
35\left(\frac{t}{T_{\mathrm{p} 1}}\right)^{4}\left(1-\frac{t}{T_{\mathrm{p} 1}}\right)^{3}+21\left(\frac{t}{T_{\mathrm{p} 1}}\right)^{5}\left(1-\frac{t}{T_{\mathrm{p} 1}}\right)^{2}+\end{cases} \\
& 7\left(\frac{t}{T_{\mathrm{p} 1}}\right)^{6}\left(1-\frac{t}{T_{\mathrm{p} 1}}\right)+\left(\frac{t}{T_{\mathrm{p} 1}}\right)^{7} \quad 0 \leq t<T_{\mathrm{p} 1} \\
& u_{\mathrm{a}}(t)=\left\{\begin{array}{l}
1 \quad T_{\mathrm{p} 1} \leq t<T_{\mathrm{p} 2} \\
\left(1-\frac{t-T_{\mathrm{p} 2}}{T_{\mathrm{p}}-T_{\mathrm{p} 2}}\right)^{7}+7\left(\frac{t-T_{\mathrm{p} 2}}{T_{\mathrm{p}}-T_{\mathrm{p} 2}}\right)\left(1-\frac{t-T_{\mathrm{p} 2}}{T_{\mathrm{p}}-T_{\mathrm{p} 2}}\right)^{6}+
\end{array}\right. \\
& 21\left(\frac{t-T_{\mathrm{p} 2}}{T_{\mathrm{p}}-T_{\mathrm{p} 2}}\right)^{2}\left(1-\frac{t-T_{\mathrm{p} 2}}{T_{\mathrm{p}}-T_{\mathrm{p} 2}}\right)^{5}+ \\
& 35\left(\frac{t-T_{\mathrm{p} 2}}{T_{\mathrm{p}}-T_{\mathrm{p} 2}}\right)\left(1-\frac{t-T_{\mathrm{p} 2}}{T_{\mathrm{p}}-T_{\mathrm{p} 2}}\right)^{6} \quad T_{\mathrm{p} 2} \leq t<T_{\mathrm{p}} \\
& 0 \quad t \geq T_{\text {p }}
\end{aligned}
$$
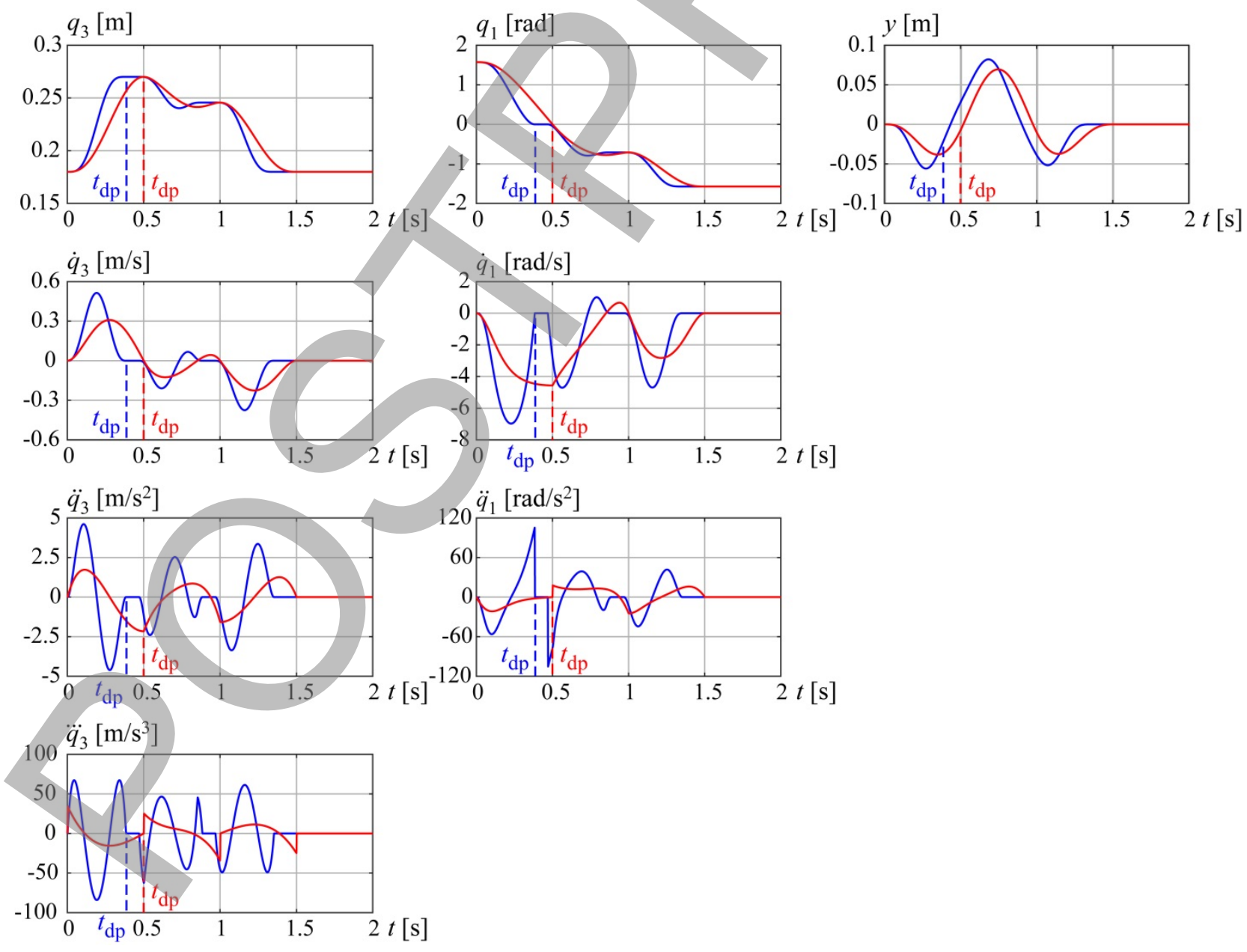

Figure 6. Input shaping, Case 1 (red line) and Case 2 (blue line): Slider $q_{3}(t)$, crank $q_{1}(t)$ motion profiles (and derivatives) and system response $y(t)$. 
Although both examples present some discontinuities in the crank angular acceleration $\ddot{q}_{1}(t)$, all values are finite and hence, it is possible to reproduce the crank coordinate $q_{1}(t)$ with a standard electric actuator, according to the criteria specified in Section 2.

\subsection{Pulse time-convolution}

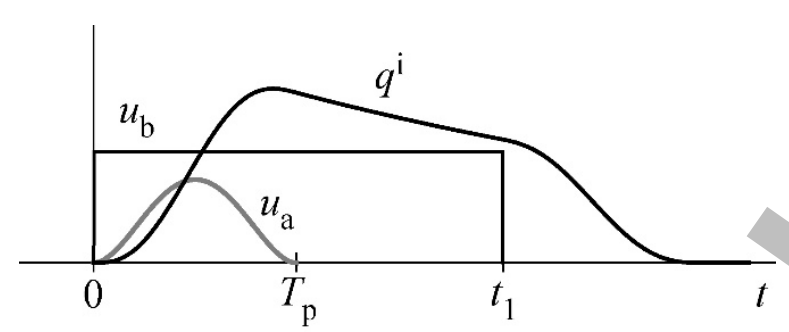

Figure 7. Pulse time-convolution method.

The second method used in this study obtains a motion profile $q^{\mathrm{i}}(t)$ by means of: $\left.i\right)$ the time-domain convolution between an arbitrary unshaped command $u_{\mathrm{a}}(t)$ and a pulse $u \mathrm{~b}(t)$ with a proper duration, and later multiplying by a negative exponential function (Figure 7), or $i i$ ) the time-domain convolution between $u_{\mathrm{a}}(t)$ and a pulse $u \mathrm{~b}(t)$, previously multiplied by a negative exponential function. These approaches are described by Equations (17) and (18), respectively.

$$
\begin{aligned}
& q^{\mathrm{i}}(t)=A\left[u_{\mathrm{a}}(t) \otimes u_{\mathrm{b}}(t)\right] \mathrm{e}^{-\zeta \omega_{0} t}+q^{\mathrm{i}}(0) \\
& q^{\mathrm{i}}(t)=A u_{\mathrm{a}}(t) \otimes\left[u_{\mathrm{b}}(t) \mathrm{e}^{-\zeta \omega_{0} t}\right]+q^{\mathrm{i}}(0)
\end{aligned}
$$

Where $A$ is a constant value and

$$
u_{\mathrm{b}}(t)= \begin{cases}0 & t \leq 0 \\ 1 & 0<t \leq t_{1} ; \quad t_{1}=\frac{2 \pi}{\omega_{\mathrm{d}}} \\ 0 & t>t_{1}\end{cases}
$$

With regard to the continuity degree, we show in Appendix that the pulse time-convolution increases the resulting function $q^{\mathrm{i}}(t)$ to $C^{n}$, assuming that $u_{\mathrm{a}}(t)$ is $C^{n-1}$ for all its domain. This assertion is valid if $u_{\mathrm{a}}(t)$ and its $n-1$ first derivatives start and finish with a null value.

The algorithm proposed is analogous to the former method. Nevertheless, in order to guarantee the dead point configuration, parameter $A$ must be calculated with Equations (20) and (21) while the motion profile is obtained using Equations (17) and (18), respectively.

$$
\begin{aligned}
& A=\frac{q^{\mathrm{i}}\left(t_{\mathrm{dp}}\right)-q^{\mathrm{i}}(0)}{\max \left(\left[u_{\mathrm{a}}(t) \otimes u_{\mathrm{b}}(t)\right] \mathrm{e}^{-\zeta \omega_{\mathrm{b}} t}\right)} \\
& A=\frac{q^{\mathrm{i}}\left(t_{\mathrm{dp}}\right)-q^{\mathrm{i}}(0)}{\max \left(u_{\mathrm{a}}(t) \otimes\left[u_{\mathrm{b}}(t) \mathrm{e}^{-\zeta \omega_{0} t}\right]\right)}
\end{aligned}
$$




\section{Examples}

Pulse time-convolution, Case $1\left(\dot{q}^{\mathrm{i}}\left(t_{\mathrm{dp}}\right)=0\right.$ and $\left.\ddot{q}^{\mathrm{i}}\left(t_{\mathrm{dp}}\right) \neq 0\right)$ :

Figure 8 (red line) shows an example of the slider coordinate $q_{3}(t)$, which follows a displacement profile $C^{2}$, and its corresponding crank angular coordinate $q_{1}(t)$. The profile $u_{\mathrm{a}}(t)$ is a Bézier curve with $g=4, p=5$ and a total duration $T_{\mathrm{p}}=0.7 \mathrm{~s}$, described by Equation (22). The oscillatory system used is the same as for the previous examples.

$$
u_{\mathrm{a}}(t)=\left\{\begin{array}{l}
0 \quad t<0 \\
\left(\frac{t}{T_{\mathrm{p}}}\right)^{2}\left(1-\frac{t}{T_{\mathrm{p}}}\right)^{2} \quad 0 \leq t<T_{\mathrm{p}} \\
0 \quad t \geq T_{\mathrm{p}}
\end{array}\right.
$$

Pulse time-convolution, Case $2\left(\dot{q}^{\mathrm{i}}\left(t_{\mathrm{dp}}\right)=0, \ddot{q}^{\mathrm{i}}\left(t_{\mathrm{dp}}\right)=0\right.$ and $\left.\ddot{q}^{\mathrm{i}}\left(t_{\mathrm{dp}}\right)=0\right)$ :

Figure 8 (blue line) shows an analogous example to the previous one, where $q_{3}(t)$ follows a displacement profile $C^{3}$ and $u_{\mathrm{a}}(t)$ is a symmetric piecewise profile made of Bézier curves $(g=5, p=6)$ and constant functions, multiplied by an exponential timefunction, as indicated by Equation (23) $\left(T_{\mathrm{p} 1}=0.4 \mathrm{~s}, T_{\mathrm{p} 2}=1.6 \mathrm{~s}\right.$ and $\left.T_{\mathrm{p}}=2.0 \mathrm{~s}\right)$.

$$
u_{\mathrm{a}}(t)=\mathrm{e}^{\zeta \omega_{0} t} \cdot\left\{\begin{array}{l}
10\left(\frac{t}{T_{\mathrm{p} 1}}\right)^{3}\left(1-\frac{t}{T_{\mathrm{p} 1}}\right)^{2}+5\left(\frac{t}{T_{\mathrm{p} 1}}\right)^{4}\left(1-\frac{t}{T_{\mathrm{p} 1}}\right)+\left(\frac{t}{T_{\mathrm{p} 1}}\right)^{5} \quad 0 \leq t<T_{\mathrm{p} 1} \\
\left(1-\frac{t-T_{\mathrm{p} 2}}{T_{\mathrm{p}}-T_{\mathrm{p} 2}}\right)^{5}+5\left(\frac{t-T_{\mathrm{p} 2}}{T_{\mathrm{p}}-T_{\mathrm{p} 2}}\right)\left(1-\frac{t-T_{\mathrm{p} 2}}{T_{\mathrm{p}}-T_{\mathrm{p} 2}}\right)^{4}+ \\
10\left(\frac{t-T_{\mathrm{p} 2}}{T_{\mathrm{p}}-T_{\mathrm{p} 2}}\right)^{2}\left(1-\frac{t-T_{\mathrm{p} 2}}{T_{\mathrm{p}}-T_{\mathrm{p} 2}}\right)^{3} \quad T_{\mathrm{p} 2} \leq t<T_{\mathrm{p}}
\end{array}\right.
$$

The results in both cases are analogous to the ones in the input shaper method. Although some discontinuities are shown in the crank angular acceleration $\ddot{q}_{1}(t)$, both motion profiles $q_{1}(t)$ can be reproduced by standard electric actuators. 

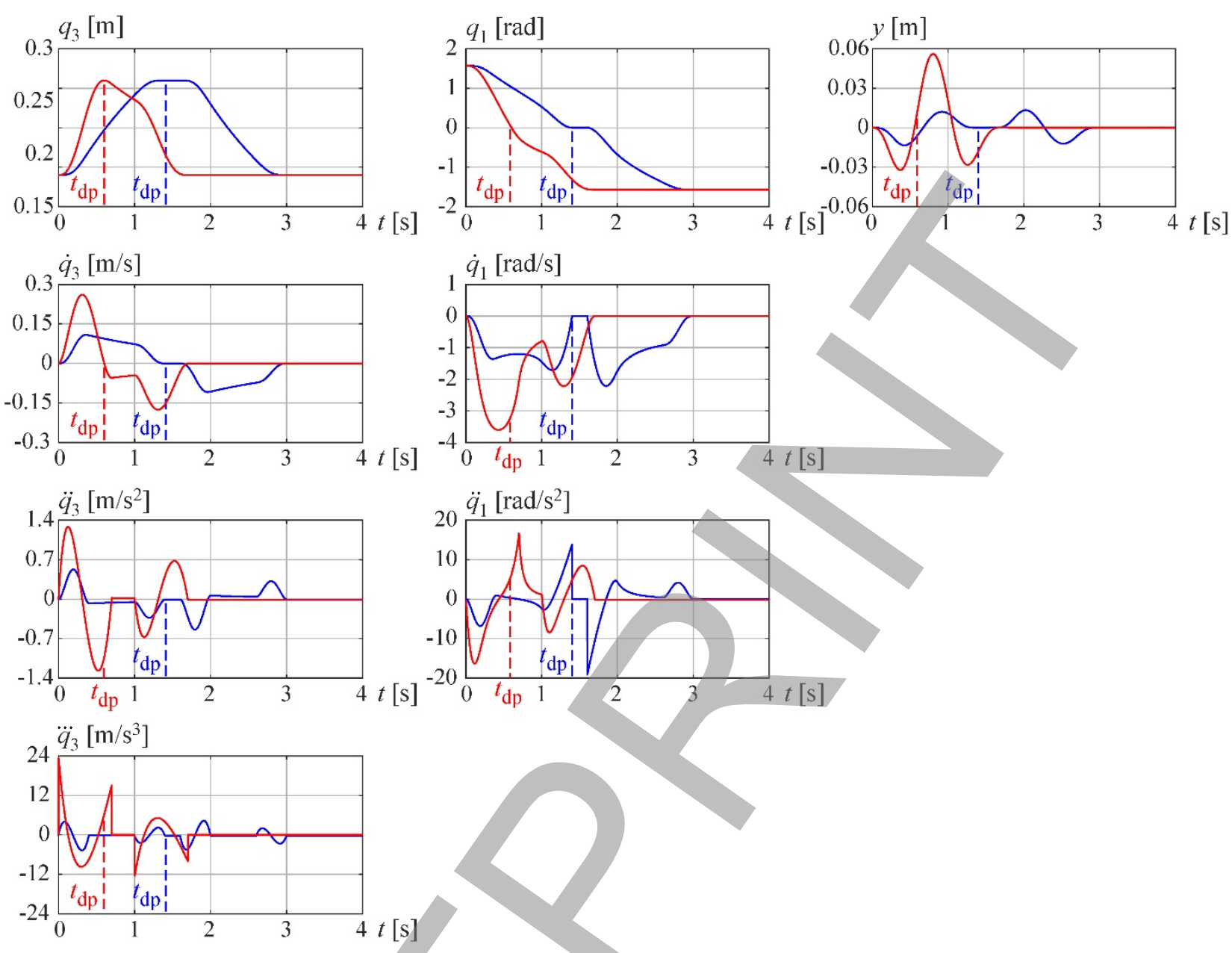

Figure 8. Pulse time-convolution, Case 1 (red line) and Case 2 (blue line): Slider $q_{3}(t)$, crank $q_{1}(t)$ motion profiles (and derivatives) and system response $y(t)$.

\section{Experimental results}

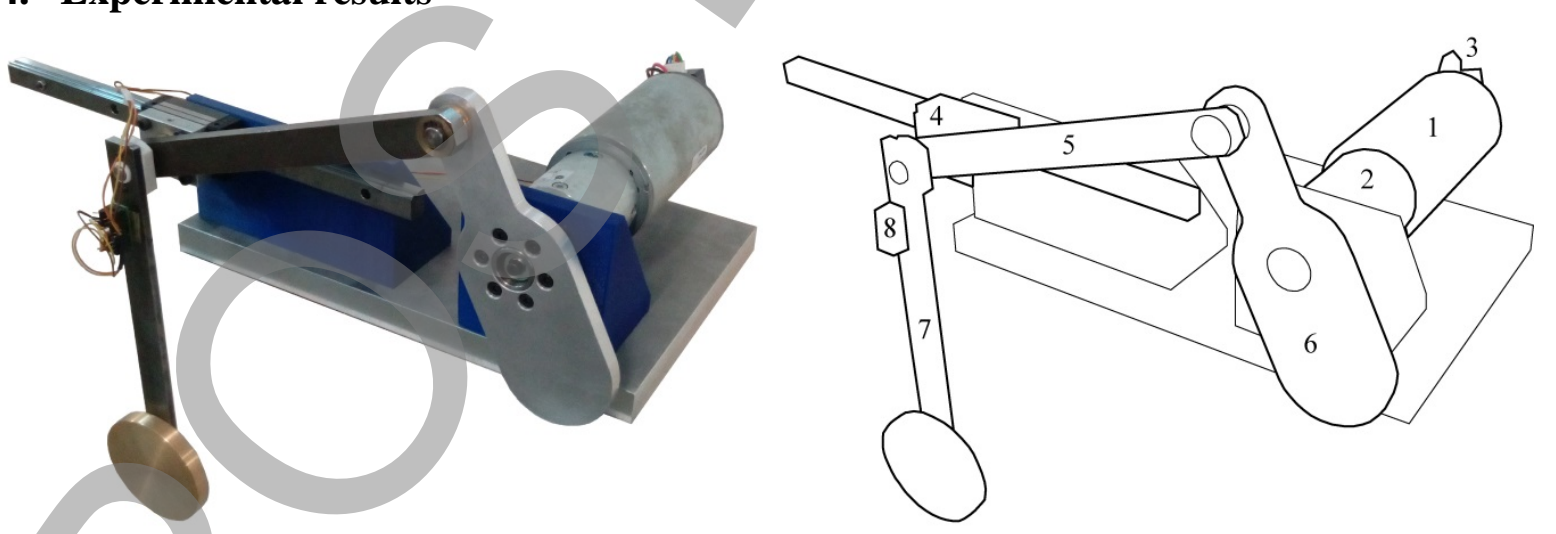

Figure 9. Test bed and components: 1) motor, 2) gear reducer, 3) encoder, 4) slider, 5) rod, 6) crank, 7) pendulum, and 8) gyroscope.

A slider-crank mechanism was built to validate the effectiveness of the proposed method (Figure 9). As a vibratory system, we used a pendulum whose axis of rotation moves together with the slider. The angle of the crank follows an input command, driven through a control, a DC motor and a planetary reducer. The feedback for the control loop is provided by a rotary incremental encoder mounted on the motor shaft. A gyroscope attached to the pendulum bar provides the measure of angular velocity $\dot{\theta}(t)$ 
and by numerical integration, we obtain the angular displacement $\theta(t)$. Table 2 presents a summary of the parameters and devices used for this test bed, according to the nomenclature of Figure 10.

\begin{tabular}{cc}
\hline Parameter/device & Value/specification \\
\hline$r$ & $75 \mathrm{~mm}$ \\
\hline$l$ & $195 \mathrm{~mm}$ \\
\hline$l_{\mathrm{p}}$ & $178 \mathrm{~mm}$ \\
\hline$m$ & $0.575 \mathrm{~kg}$ \\
\hline$I_{\mathrm{G}}$ & $2.4 \cdot 10^{-3} \mathrm{~kg} \cdot \mathrm{m}^{2}$ \\
\hline$c$ & $2.3 \cdot 10^{-3} \mathrm{~N} \cdot \mathrm{m} /(\mathrm{rad} / \mathrm{s})$ \\
\hline$f_{\mathrm{d}}$ & $1.135 \mathrm{~Hz}$ \\
\hline$\zeta$ & 0.008 \\
\hline Motor & Permanent magnet DC brushed, 24V \\
\hline Gear reducer & Planetary with $20.25 \mathrm{ratio}$ \\
\hline Encoder & Rotary, 500 cycles per revolution \\
\hline Gyroscope & ADXR300, $\pm 300 \%$ s, $5 \mathrm{mV} /(\% / \mathrm{s})$ Low pass \\
& filter RC, cutoff freq. at $40 \mathrm{~Hz}$ \\
\hline
\end{tabular}

Table 2. Test bed parameters and specifications.

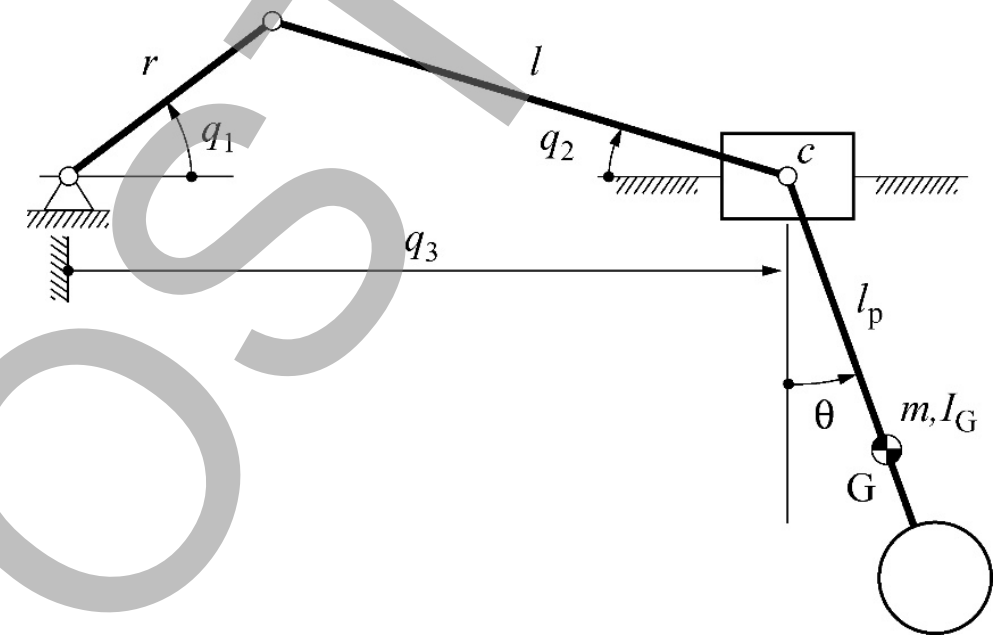

Figure 10. Test bed sketch.

The motion equation of the pendulum described in Figure 10 for a given $q_{3}(t)$ can be obtained by

$$
\left(m l_{\mathrm{p}}^{2}+I_{\mathrm{G}}\right) \ddot{\theta}+c \dot{\theta}+m \mathrm{~g} l_{\mathrm{p}} \sin \theta=-m \ddot{q}_{3}(t) l_{\mathrm{p}} \cos \theta
$$

If we assume small oscillations around $\theta=0^{\circ}$, during and after the transient, Equation (24) can be linearized as 


$$
\left(m l_{\mathrm{p}}^{2}+I_{\mathrm{G}}\right) \ddot{\theta}+c \dot{\theta}+m \mathrm{~g} l_{\mathrm{p}} \theta=-m \ddot{q}_{3}(t) l_{\mathrm{p}}
$$

Rewriting in a canonical form, it yields

$$
\ddot{\theta}+2 \zeta \omega_{0} \dot{\theta}+\omega_{0}^{2} \theta=-\frac{\omega_{0}^{2}}{\mathrm{~g}} \ddot{q}_{3}(t)
$$

where the system's natural frequency and the damping ratio are, respectively

$$
\begin{gathered}
\omega_{0}=\sqrt{\frac{m \mathrm{~g} l_{\mathrm{p}}}{m l_{\mathrm{p}}^{2}+I_{\mathrm{G}}}} \\
\zeta=\frac{c}{2 \sqrt{m \mathrm{~g} l_{\mathrm{p}}\left(m l_{\mathrm{p}}^{2}+I_{\mathrm{G}}\right)}}
\end{gathered}
$$

Notice that this system, assuming small oscillations, is equivalent to the one depicted in Figure 2.

We obtain the pendulum oscillation frequency $f_{\mathrm{d}}=\omega_{\mathrm{d}} /(2 \pi)$ and the damping ratio $\zeta$ by direct measurement. The bandwidths of the motor-gear reducer-system and control electronics are far from the pendulum's natural frequency and therefore the filtering effect of the input command is expected to be minimal.

Two series, which each include three tests, were carried out. Both series set the initial condition of the slider coordinate at $q_{3}=180 \mathrm{~mm}$, which means a crank position of $q_{1}=90^{\circ}$, moving through the dead point located at $q_{3}=270 \mathrm{~mm}$ to a final coordinate of $q_{3}=180 \mathrm{~mm}$, at $q_{1}=-90^{\circ}$.

Series $a)$ :

The three tests have a motion profile with a total duration of $1.44 \mathrm{~s}$ and include the methods:

i) Unshaped command alone $u_{\mathrm{a}}(t)$ described by Equation $(15)$ with $T_{\mathrm{p}}=1.44 \mathrm{~s}$ that, according to the requirements pointed out in Section 2, has a minimum continuity of $C^{2}$ to be reproduced by conventional electric actuators.

ii) Input shaper (Smith [4] and Singer and Seering [5]) that uses the unshaped command $u_{\mathrm{a}}(t)$ mentioned above with $T_{\mathrm{p}}=1.0 \mathrm{~s}$.

iii) Pulse time-convolution (Veciana and Cardona [18]). Equation (22) with $T_{\mathrm{p}}=0.560 \mathrm{~s}$ describes the unshaped command used in this case, which is $C^{1}$ according to the stated requirements.

Figure 11, $a$ ) depicts the slider displacement $q_{3}(t)$, and the crank and pendulum real angular velocities, $\dot{q}_{1}(t)$ and $\dot{\theta}(t)$, respectively, for each of the aforementioned methods. Video 1, Video 2, and Video 3 show them, respectively (electronic version only). Notice the important amplitude of the residual vibration of $\dot{\theta}(t)$ in the first test, where no shaper is used, compared with the virtually null residual vibrations of the second and third tests. Moreover, the goodness of the obtained experimental results, together with the simulations of section 3, validate the role of the proposed algorithm. It provides a method to design a motion profile $q_{3}(t)$ to reduce residual vibrations. The algorithm also guarantees the feasibility of the motion profile, avoiding any infinite 
discontinuity in the acceleration of the driven coordinate $q_{1}(t)$. Therefore, according to the hypotheses formulated in section 2.1 , the mechanism can be driven with a standard electric actuator.
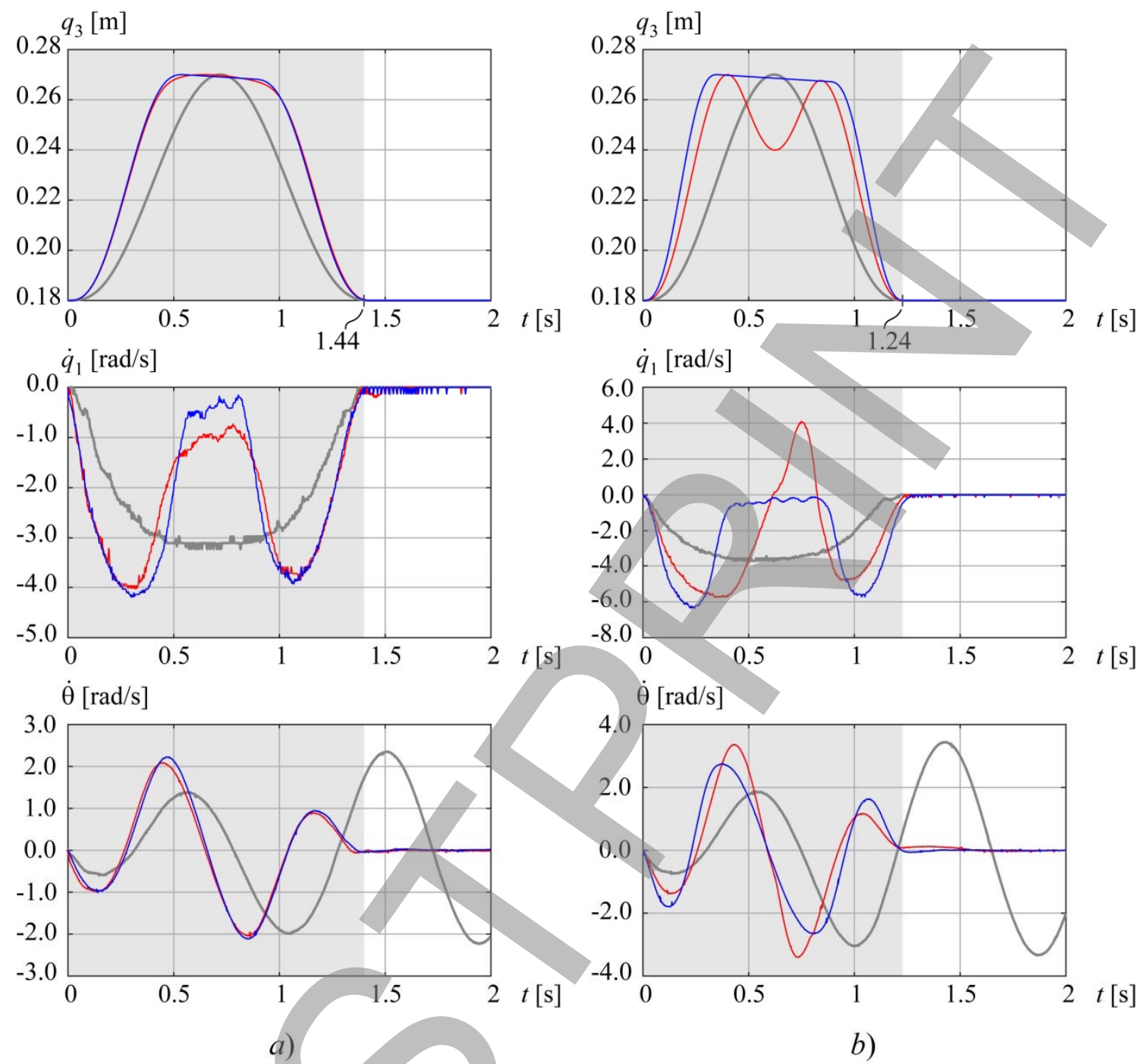

Figure 11. Test results: unshaped command (grey line), input shaper (red line) and pulse time-convolution (blue line) for test series: $a$ ) $1.44 \mathrm{~s}$ input total duration and $b$ ) $1.24 \mathrm{~s}$ input total duration.

\section{Test series $a$ ): Unshaped command alone}

Video 1. (Electronic version only) Test series $a$ ): Unshaped command alone. 


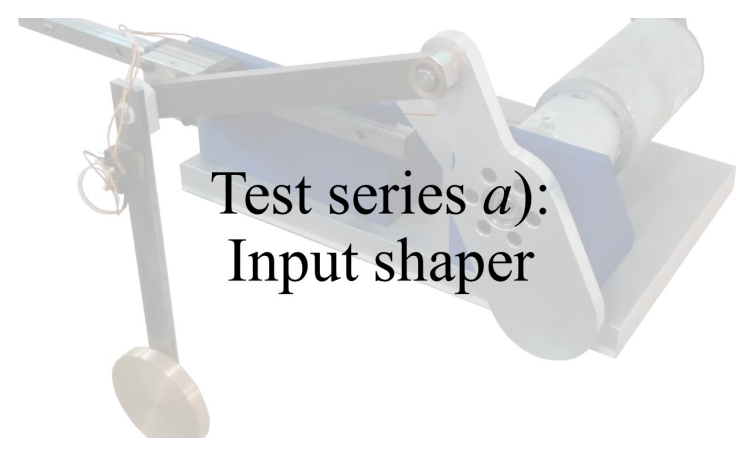

Video 2. (Electronic version only) Test series $a$ ): Input shaper.

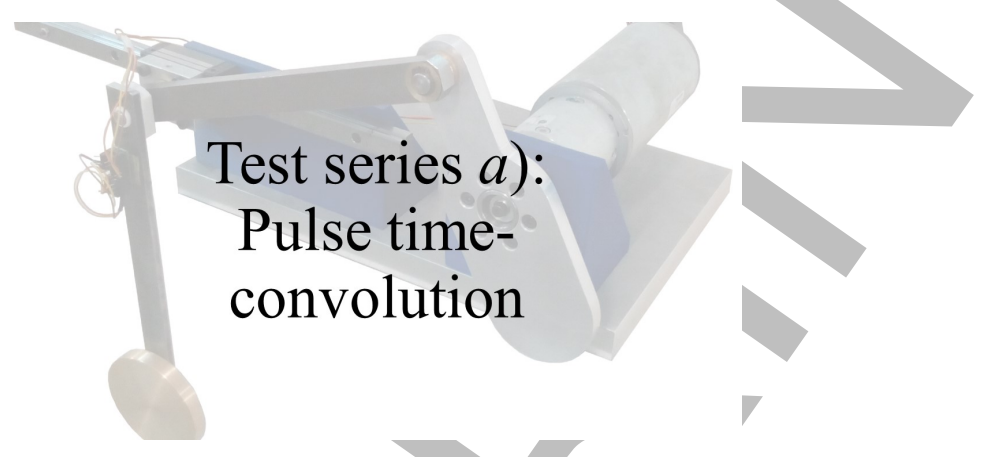

Video 3. (Electronic version only) Test series a): Pulse time-convolution.

Series $b$ ):

In this case, the three tests have a motion profile with a total duration of $1.24 \mathrm{~s}$ and include the same methods as the ones in Series a). Figure 11,b) depicts the time evolution of the slider displacement, and the crank and pendulum real angular velocities as well. Video 4, Video 5, and Video 6 show these tests, respectively (electronic version only). Again, notice the high amplitude of the residual vibration of $\dot{\theta}(t)$ in the first test, where no method is used. The second and third tests have, as expected, a virtually null residual vibration. Additionally, the crank angular velocity shows no sign change in the pulse time-convolution technique, compared with the input shaper, which means a minimum-acceleration-switch shape. This is translated to a smoother motion profile that avoids unwanted dynamics that can be caused by transmission backlash, mechanical plays, or electronic hysteresis [18]. As mentioned in series $a$ ) the proposed algorithm ensures the feasibility of $q_{3}(t)$. The effectiveness in the reduction of the residual vibration observed in the experimental process, together with the simulations carried out, validate its benefit.

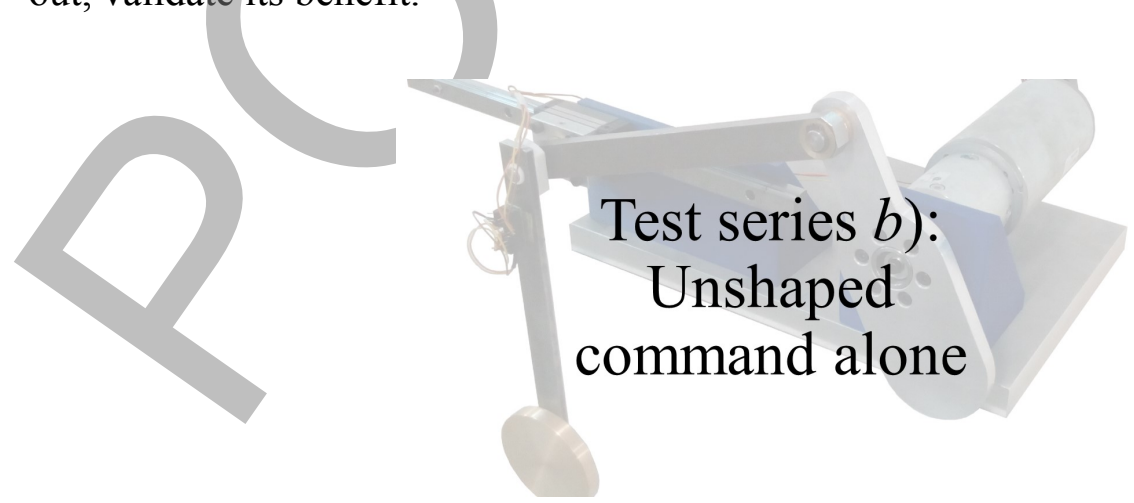

Video 4. (Electronic version only) Test series $b$ ): Unshaped command alone. 


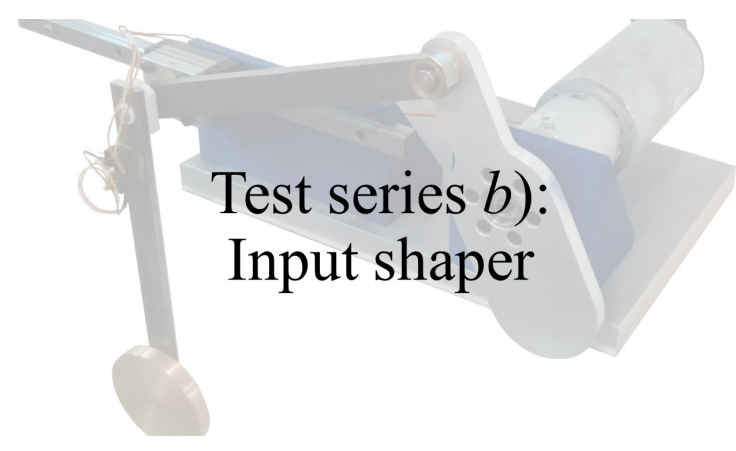

Video 5. (Electronic version only) Test series $b$ ): Input shaper.

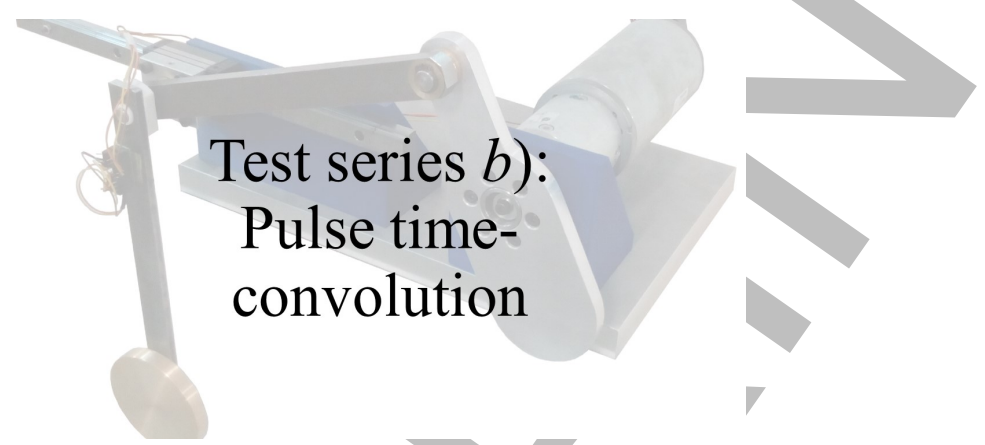

Video 6. (Electronic version only) Test series b): Pulse time-convolution.

\section{Conclusions}

This study proposes the reduction of residual vibrations in one degree-of-freedom oscillatory systems, when they are driven by an arbitrary linkage mechanism working through a dead point configuration. The development has been focused on a slider-crank mechanism. Nevertheless, the method can be easily extended to other one degree-of-freedom linkage mechanisms.

The proposed algorithm guarantees both, the feasibility of the motion profile with regard to the limitations mentioned in [22] and [23], and the effectiveness in the reduction of residual vibrations. The development is focused on two methods: input shaping and pulse time-convolution. Some examples of these profiles are presented and the results of the simulations carried out validate the algorithm.

Furthermore, this algorithm is supported by the analysis shown in the Appendix, in which the effect on the continuity degree of the time convolution operation for those two methods is introduced. Basically, the authors conclude that the first method maintains the continuity degree from the unshaped command $u_{\mathrm{a}}(t)$, and the second method increases it by one degree. In addition, it is proven that the multiplication by an exponential time function does not modify the continuity degree.

Finally, the virtually null residual vibrations of the presented experimental results validate the algorithm in a real scenario.

\section{Appendix}

In Section 3, we explained that the continuity degree of the resulting signal $q^{\mathrm{i}}(t)$ remains invariable with regard to $u_{\mathrm{a}}(t)$ when the input shaping method is used, and increases by one degree if the pulse time-convolution is chosen. Herein those statements are proven. Additionally, we demonstrate that multiplying an arbitrary signal by a negative exponential time function does not affect its continuity degree. 
Assume that $T_{\mathrm{p}}$ is the total duration of the unshaped function $u_{\mathrm{a}}(t)$ and that the oscillation frequency of the oscillatory system is $f_{\mathrm{d}}$. The relative duration between $T_{\mathrm{p}}$ and the oscillation period $1 / f_{\mathrm{d}}$ brings about different analyses, which are shown below.

\subsection{Input shaping}

In this section, we study the continuity of the time convolution between an arbitrary unshaped command $u_{\mathrm{a}}(t)$ and a sequence of two impulses $u_{\mathrm{b}}(t)$. The impulses are located at a relative time of $1 /\left(2 f_{\mathrm{d}}\right)$ between them, which leads to two different cases depending on the comparison between $T_{\mathrm{p}}$ and half of the oscillation period $1 /\left(2 f_{\mathrm{d}}\right)$.

In this development, we assume that $u_{\mathrm{a}}(t)$ has a minimum continuity degree $C^{n}$ for all of its domain (including the junctions of the different intervals in which the analytical piecewise function is split).

a) $T_{\mathrm{p}} \geq 1 /\left(2 f_{\mathrm{d}}\right)$

Figure 12 shows the different intervals in which the time convolution operation can be divided. The resulting piecewise function $q^{\mathrm{i}}(t)$ is analytically described in Equation (a.1), and is obtained with the general formulation of Equation (11).

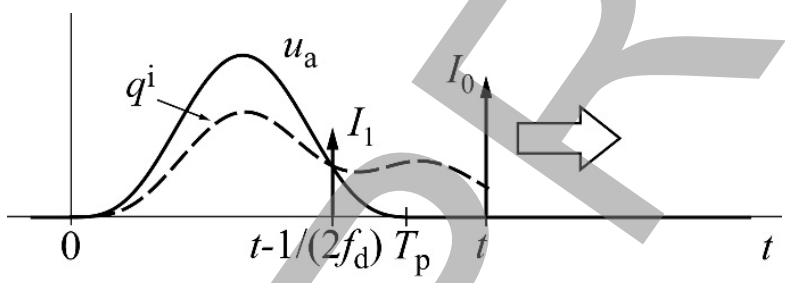

Figure 12. Evolution of the convolution in the time domain of an unshaped command $u_{\mathrm{a}}(t)$ with an impulse sequence.

$$
q^{\mathrm{i}}(t)= \begin{cases}0 & t \leq 0 \\ I_{0} u_{\mathrm{a}}(t) & 0<t \leq 1 /\left(2 f_{\mathrm{d}}\right) \\ I_{0} u_{\mathrm{a}}(t)+I_{1} u_{\mathrm{a}}\left(t-1 /\left(2 f_{\mathrm{d}}\right)\right) & 1 /\left(2 f_{\mathrm{d}}\right)<t \leq T_{\mathrm{p}} \\ I_{1} u_{\mathrm{a}}\left(t-1 /\left(2 f_{\mathrm{d}}\right)\right) & T_{\mathrm{p}}<t \leq T_{\mathrm{p}}+1 /\left(2 f_{\mathrm{d}}\right) \\ 0 & T_{\mathrm{p}}+1 /\left(2 f_{\mathrm{d}}\right)<t\end{cases}
$$

In order to study the minimum continuity degree of $q^{\mathrm{i}}(t)$, Equation (a.1) is $n$-times differentiated. 


$$
\begin{gathered}
\dot{q}^{\mathrm{i}}(t)= \begin{cases}0 & t \leq 0 \\
I_{0} \dot{u}_{\mathrm{a}}(t) & 0<t \leq 1 /\left(2 f_{\mathrm{d}}\right) \\
I_{0} \dot{u}_{\mathrm{a}}(t)+I_{1} \dot{u}_{\mathrm{a}}\left(t-1 /\left(2 f_{\mathrm{d}}\right)\right) & 1 /\left(2 f_{\mathrm{d}}\right)<t \leq T_{\mathrm{p}} \\
I_{1} \dot{u}_{\mathrm{a}}\left(t-1 /\left(2 f_{\mathrm{d}}\right)\right) & T_{\mathrm{p}}<t \leq T_{\mathrm{p}}+1 /\left(2 f_{\mathrm{d}}\right) \\
0 & T_{\mathrm{p}}+1 /\left(2 f_{\mathrm{d}}\right)<t\end{cases} \\
q^{\mathrm{i}(n)}(t)
\end{gathered}
$$

In Equation (a.3) we impose the condition that $q^{\mathrm{i}}(t)$, as well as the first $n$ derivatives, $\dot{q}^{\mathrm{i}}(t), \ddot{q}^{\mathrm{i}}(t) \ldots, q^{\mathrm{i}(n)}(t)$, are continuous at the time instants $t=0, t=1 /\left(2 f_{\mathrm{d}}\right), t=T_{\mathrm{p}}$, and $t=T_{\mathrm{p}}+1 /\left(2 f_{\mathrm{d}}\right)$.

$$
\begin{array}{ll}
0=I_{0} u_{\mathrm{a}}\left(0^{+}\right) & t=0 \\
I_{0} u_{\mathrm{a}}\left(1 /\left(2 f_{\mathrm{d}}\right)^{-}\right)=I_{0} u_{\mathrm{a}}\left(1 /\left(2 f_{\mathrm{d}}\right)^{+}\right)+I_{1} u_{\mathrm{a}}\left(0^{+}\right) & t=1 /\left(2 f_{\mathrm{d}}\right) \\
I_{0} u_{\mathrm{a}}\left(T_{\mathrm{p}}^{-}\right)+I_{1} u_{\mathrm{a}}\left(T_{\mathrm{p}}^{-}-1 /\left(2 f_{\mathrm{d}}\right)\right)=I_{1} u_{\mathrm{a}}\left(T_{\mathrm{p}}^{+}-1 /\left(2 f_{\mathrm{d}}\right)\right) & t=T_{\mathrm{p}} \\
I_{1} u_{\mathrm{a}}\left(T_{\mathrm{p}}^{-}\right)=0 & t=T_{\mathrm{p}}+1 /\left(2 f_{\mathrm{d}}\right) \\
& \\
0=I_{0} \dot{u}_{\mathrm{a}}\left(0^{+}\right) & t=0 \\
I_{0} \dot{u}_{\mathrm{a}}\left(1 /\left(2 f_{\mathrm{d}}\right)^{-}\right)=I_{0} \dot{u}_{\mathrm{a}}\left(1 /\left(2 f_{\mathrm{d}}\right)^{+}\right)+I_{1} \dot{u}_{\mathrm{a}}\left(0^{+}\right) & t=1 /\left(2 f_{\mathrm{d}}\right) \\
I_{0} \dot{u}_{\mathrm{a}}\left(T_{\mathrm{p}}^{-}\right)+I_{1} \dot{u}_{\mathrm{a}}\left(T_{\mathrm{p}}^{-}-1 /\left(2 f_{\mathrm{d}}\right)\right)=I_{1} \dot{u}_{\mathrm{a}}\left(T_{\mathrm{p}}^{+}-1 /\left(2 f_{\mathrm{d}}\right)\right) & t=T_{\mathrm{p}} \\
I_{1} \dot{u}_{\mathrm{a}}\left(T_{\mathrm{p}}^{-}\right)=0 & t=T_{\mathrm{p}}+1 /\left(2 f_{\mathrm{d}}\right) \\
\quad \vdots & t=0 \\
0=I_{0} u_{\mathrm{a}}^{(n)}\left(0^{+}\right) & t=1 /\left(2 f_{\mathrm{d}}\right) \\
I_{0} u_{\mathrm{a}}^{(n)}\left(1 /\left(2 f_{\mathrm{d}}\right)^{-}\right)=I_{0} u_{\mathrm{a}}^{(n)}\left(1 /\left(2 f_{\mathrm{d}}\right)^{+}\right)+I_{1} u_{\mathrm{a}}^{(n)}\left(0^{+}\right) & \\
I_{0} u_{\mathrm{a}}^{(n)}\left(T_{\mathrm{p}}^{-}\right)+I_{1} u_{\mathrm{a}}^{(n)}\left(T_{\mathrm{p}}^{-}-1 /\left(2 f_{\mathrm{d}}\right)\right)=I_{1} u_{\mathrm{a}}^{(n)}\left(T_{\mathrm{p}}^{+}-1 /\left(2 f_{\mathrm{d}}\right)\right) & t=T_{\mathrm{p}} \\
I_{1} u_{\mathrm{a}}^{(n)}\left(T_{\mathrm{p}}^{-}\right)=0 & t=T_{\mathrm{p}}+1 /\left(2 f_{\mathrm{d}}\right)
\end{array}
$$

If $u_{\mathrm{a}}(t)$ is $C^{n}$ for all its domain, the following results: 


$$
\begin{aligned}
& u_{\mathrm{a}}\left(1 /\left(2 f_{\mathrm{d}}\right)^{-}\right)=u_{\mathrm{a}}\left(1 /\left(2 f_{\mathrm{d}}\right)^{+}\right) \\
& u_{\mathrm{a}}\left(T_{\mathrm{p}}^{-}-1 /\left(2 f_{\mathrm{d}}\right)\right)=u_{\mathrm{a}}\left(T_{\mathrm{p}}^{+}-1 /\left(2 f_{\mathrm{d}}\right)\right) \\
& \dot{u}_{\mathrm{a}}\left(1 /\left(2 f_{\mathrm{d}}\right)^{-}\right)=\dot{u}_{\mathrm{a}}\left(1 /\left(2 f_{\mathrm{d}}\right)^{+}\right) \\
& \dot{u}_{\mathrm{a}}\left(T_{\mathrm{p}}^{-}-1 /\left(2 f_{\mathrm{d}}\right)\right)=\dot{u}_{\mathrm{a}}\left(T_{\mathrm{p}}^{+}-1 /\left(2 f_{\mathrm{d}}\right)\right) \\
& \vdots \\
& u_{\mathrm{a}}^{(n)}\left(1 /\left(2 f_{\mathrm{d}}\right)^{-}\right)=u_{\mathrm{a}}^{(n)}\left(1 /\left(2 f_{\mathrm{d}}\right)^{+}\right) \\
& u_{\mathrm{a}}^{(n)}\left(T_{\mathrm{p}}^{-}-1 /\left(2 f_{\mathrm{d}}\right)\right)=u_{\mathrm{a}}^{(n)}\left(T_{\mathrm{p}}^{+}-1 /\left(2 f_{\mathrm{d}}\right)\right)
\end{aligned}
$$

By introducing this result into Equation (a.3), it yields:

$$
\begin{aligned}
& u_{\mathrm{a}}\left(0^{+}\right)=\dot{u}_{\mathrm{a}}\left(0^{+}\right) \ldots=u_{\mathrm{a}}^{(n)}\left(0^{+}\right)=0 \\
& u_{\mathrm{a}}\left(T_{\mathrm{p}}^{-}\right)=\dot{u}_{\mathrm{a}}\left(T_{\mathrm{p}}^{-}\right) \ldots=u_{\mathrm{a}}^{(n)}\left(T_{\mathrm{p}}^{-}\right)=0
\end{aligned}
$$

Conclusion $A$ : $q^{\mathrm{i}}(t)$ has at least a continuity degree of $C^{n}$ if $u_{\mathrm{a}}(t)$ is $C^{n}$ for its entire domain, and $u_{\mathrm{a}}(t)$ and its $n$ first derivatives start and finish with a null value.

b) $T_{\mathrm{p}}<1 /\left(2 f_{\mathrm{d}}\right)$

In this case, the different intervals of the analytical function can be described by Equation (a.6). As in the former case, this function has been obtained by using Equation (11).

$$
q^{\mathrm{i}}(t)= \begin{cases}0 & t \leq 0 \\ I_{0} u_{\mathrm{a}}(t) & 0<t \leq T_{\mathrm{p}} \\ 0 & T_{\mathrm{p}}<t \leq 1 /\left(2 f_{\mathrm{d}}\right) \\ I_{1} u_{\mathrm{a}}\left(t-1 /\left(2 f_{\mathrm{d}}\right)\right) & 1 /\left(2 f_{\mathrm{d}}\right)<t \leq T_{\mathrm{p}}+1 /\left(2 f_{\mathrm{d}}\right) \\ 0 & T_{\mathrm{p}}+1 /\left(2 f_{\mathrm{d}}\right)<t\end{cases}
$$

As in the previous case and following the same procedure, the same condition described in Conclusion $A$ can analogously be proven when the duration $T_{\mathrm{p}}$ of $u_{\mathrm{a}}(t)$ is smaller than half of the oscillation period, $1 /\left(2 f_{\mathrm{d}}\right)$.

\subsection{Pulse time-convolution}

In this case, the continuity is analyzed when the time convolution is carried out between an arbitrary unshaped command $u_{\mathrm{a}}(t)$ and a rectangular pulse $u \mathrm{~b}(t)$ of amplitude $A_{\mathrm{d}}$ and duration $1 / f_{\mathrm{d}}$. This analysis is also split into two different cases depending on the relative duration between $T_{\mathrm{p}}$ and the oscillation period $1 / f_{\mathrm{d}}$.

We assume now that $u_{\mathrm{a}}(t)$ has a minimum continuity degree $C^{n-1}$ for its entire domain, which includes junctions between piecewise functions, as well as for the former case.

a) $T_{\mathrm{p}} \geq 1 / f_{\mathrm{d}}$

Figure 13 points out the different intervals of the function $q^{\mathrm{i}}(t)$ that results from the time convolution between $u \mathrm{a}(t)$ and $u \mathrm{~b}(t)$. Equation (a.7) describes the analytical expression of this piecewise function, obtained from the time-domain convolution detailed in Equation (17). 


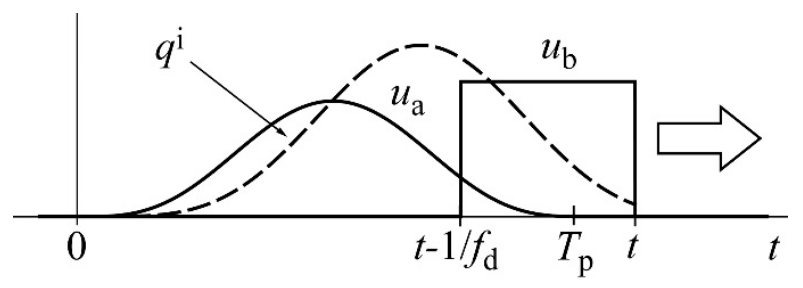

Figure 13. Evolution of the convolution in the time domain of an unshaped command $u_{\mathrm{a}}(t)$ with a pulse.

$$
q^{\mathrm{i}}(t)= \begin{cases}0 & t \leq 0 \\ A_{\mathrm{d}} \int_{0}^{t} u_{\mathrm{a}}(\tau) \mathrm{d} \tau & 0<t \leq 1 / f_{\mathrm{d}} \\ A_{\mathrm{d}} \int_{t-1 / f_{\mathrm{d}}}^{t} u_{\mathrm{a}}(\tau) \mathrm{d} \tau & 1 / f_{\mathrm{d}}<t \leq T_{\mathrm{p}} \\ A_{\mathrm{d}} \int_{t-1 / f_{\mathrm{d}}}^{T_{\mathrm{p}}} u_{\mathrm{a}}(\tau) \mathrm{d} \tau & T_{\mathrm{p}}<t \leq T_{\mathrm{p}}+1 / f_{\mathrm{d}} \\ 0 & T_{\mathrm{p}}+1 / f_{\mathrm{d}}<t\end{cases}
$$

Equation (a.7) is $n$-times differentiated in order to study the minimum continuity degree of $q^{\mathrm{i}}(t)$.

$$
\begin{aligned}
& \dot{q}^{\mathrm{i}}(t)= \begin{cases}0 & t \leq 0 \\
A_{\mathrm{d}} u_{\mathrm{a}}(t) & 0<t \leq 1 / f_{\mathrm{d}} \\
A_{\mathrm{d}}\left(u_{\mathrm{a}}(t)-u_{\mathrm{a}}\left(t-1 / f_{\mathrm{d}}\right)\right) & 1 / f_{\mathrm{d}}<t \leq T_{\mathrm{p}} \\
-A_{\mathrm{d}} u_{\mathrm{a}}\left(t-1 / f_{\mathrm{d}}\right) & T_{\mathrm{p}}<t \leq T_{\mathrm{p}}+1 / f_{\mathrm{d}} \\
0 & T_{\mathrm{p}}+1 / f_{\mathrm{d}}<t\end{cases} \\
& \ddot{q}^{\mathrm{i}}(t)= \begin{cases}0 & t \leq 0 \\
A_{\mathrm{d}} \dot{u}_{\mathrm{a}}(t) & 0<t \leq 1 / f_{\mathrm{d}} \\
A_{\mathrm{d}}\left(\dot{u}_{\mathrm{a}}(t)-\dot{u}_{\mathrm{a}}\left(t-1 / f_{\mathrm{d}}\right)\right) & 1 / f_{\mathrm{d}}<t \leq T_{\mathrm{p}} \\
-A_{\mathrm{d}} \dot{u}_{\mathrm{a}}\left(t-1 / f_{\mathrm{d}}\right) & T_{\mathrm{p}}<t \leq T_{\mathrm{p}}+1 / f_{\mathrm{d}} \\
0 & T_{\mathrm{p}}+1 / f_{\mathrm{d}}<t\end{cases} \\
& q^{\mathrm{i}(n)}(t)= \begin{cases}0 & t \leq 0 \\
A_{\mathrm{d}} u_{\mathrm{a}}^{(n-1)}(t) & 0<t \leq 1 / f_{\mathrm{d}} \\
A_{\mathrm{d}}\left(u_{\mathrm{a}}^{(n-1)}(t)-u_{\mathrm{a}}^{(n-1)}\left(t-1 / f_{\mathrm{d}}\right)\right) & 1 / f_{\mathrm{d}}<t \leq T_{\mathrm{p}} \\
-A_{\mathrm{d}} u_{\mathrm{a}}^{(n-1)}\left(t-1 / f_{\mathrm{d}}\right) & T_{\mathrm{p}}<t \leq T_{\mathrm{p}}+1 / f_{\mathrm{d}} \\
0 & T_{\mathrm{p}}+1 / f_{\mathrm{d}}<t\end{cases}
\end{aligned}
$$

As in the former case, we impose the condition that $q^{\mathrm{i}}(t)$, as well as the first $n$ derivatives, are continuous at the time instants $t=0, t=1 / f_{\mathrm{d}}, t=T_{\mathrm{p}}$, and $t=T_{\mathrm{p}}+1 / f_{\mathrm{d}}$ (Equation (a.9)). 


$$
\begin{aligned}
& 0=\int_{0}^{0^{+}} u_{\mathrm{a}}(\tau) \mathrm{d} \tau \quad t=0 \\
& \int_{0}^{\left(1 / f_{\mathrm{d}}\right)^{-}} u_{\mathrm{a}}(\tau) \mathrm{d} \tau=\int_{0^{+}}^{\left(1 / f_{\mathrm{d}}\right)^{+}} u_{\mathrm{a}}(\tau) \mathrm{d} \tau \quad t=1 / f_{\mathrm{d}} \\
& \int_{T_{\mathrm{p}}^{-}-1 / f_{\mathrm{d}}}^{T_{\mathrm{a}}^{-}} u_{\mathrm{a}}(\tau) \mathrm{d} \tau=\int_{T_{\mathrm{p}}^{+}-1 / f_{\mathrm{d}}}^{T_{\mathrm{p}}} u_{\mathrm{a}}(\tau) \mathrm{d} \tau \quad t=T_{\mathrm{p}} \\
& \int_{T_{\mathrm{p}}^{-}}^{T_{\mathrm{p}}} u_{\mathrm{a}}(\tau) \mathrm{d} \tau=0 \quad t=T_{\mathrm{p}}+1 / f_{\mathrm{d}} \\
& 0=u_{\mathrm{a}}\left(0^{+}\right) \\
& u_{\mathrm{a}}\left(\left(1 / f_{\mathrm{d}}\right)^{-}\right)=u_{\mathrm{a}}\left(\left(1 / f_{\mathrm{d}}\right)^{+}\right)-u_{\mathrm{a}}\left(0^{+}\right) \\
& u_{\mathrm{a}}\left(T_{\mathrm{p}}^{-}\right)-u_{\mathrm{a}}\left(T_{\mathrm{p}}^{-}-1 / f_{\mathrm{d}}\right)=-u_{\mathrm{a}}\left(T_{\mathrm{p}}^{+}-1 / f_{\mathrm{d}}\right) \quad t=T_{\mathrm{p}} \\
& -u_{\mathrm{a}}\left(T_{\mathrm{p}}^{-}\right)=0 \\
& t=T_{\mathrm{p}}+1 / f_{\mathrm{d}} \\
& 0=\dot{u}_{\mathrm{a}}\left(0^{+}\right) \\
& \dot{u}_{\mathrm{a}}\left(\left(1 / f_{\mathrm{d}}\right)^{-}\right)=\dot{u}_{\mathrm{a}}\left(\left(1 / f_{\mathrm{d}}\right)^{+}\right)-\dot{u}_{\mathrm{a}}\left(0^{+}\right) \quad t=1 / f_{\mathrm{d}} \\
& \dot{u}_{\mathrm{a}}\left(T_{\mathrm{p}}^{-}\right)-\dot{u}_{\mathrm{a}}\left(T_{\mathrm{p}}^{-}-1 / f_{\mathrm{d}}\right)=-\dot{u}_{\mathrm{a}}\left(T_{\mathrm{p}}^{+}-1 / f_{\mathrm{d}}\right) \quad t=T_{\mathrm{p}} \\
& -\dot{u}_{\mathrm{a}}\left(T_{\mathrm{p}}^{-}\right)=0 \quad t=T_{\mathrm{p}}+1 / f_{\mathrm{d}} \\
& 0=u_{\mathrm{a}}^{(n-1)}\left(0^{+}\right) \quad t=0 \\
& u_{\mathrm{a}}^{(n-1)}\left(\left(1 / f_{\mathrm{d}}\right)^{-}\right)=u_{\mathrm{a}}^{(n-1)}\left(\left(1 / f_{\mathrm{d}}\right)^{+}\right)-u_{\mathrm{a}}^{(n-1)}\left(0^{+}\right) \quad t=1 / f_{\mathrm{d}} \\
& u_{\mathrm{a}}^{(n-1)}\left(T_{\mathrm{p}}^{-}\right)-u_{\mathrm{a}}^{(n-1)}\left(T_{\mathrm{p}}^{-}-1 / f_{\mathrm{d}}\right)=-u_{\mathrm{a}}^{(n-1)}\left(T_{\mathrm{p}}^{+}-1 / f_{\mathrm{d}}\right) \quad t=T_{\mathrm{p}} \\
& -u_{\mathrm{a}}^{(n-1)}\left(T_{\mathrm{p}}^{-}\right)=0 \\
& t=T_{\mathrm{p}}+1 / f_{\mathrm{d}}
\end{aligned}
$$

Therefore, assuming that $u_{\mathrm{a}}(t)$ is $C^{n-1}$ inside the domain:

$$
\begin{gathered}
\dot{u}_{\mathrm{a}}\left(\left(1 / f_{\mathrm{d}}\right)^{-}\right)=\dot{u}_{\mathrm{a}}\left(\left(1 / f_{\mathrm{d}}\right)^{+}\right) \\
\dot{u}_{\mathrm{a}}\left(T_{\mathrm{p}}^{-}-1 / f_{\mathrm{d}}\right)=\dot{u}_{\mathrm{a}}\left(T_{\mathrm{p}}^{+}-1 / f_{\mathrm{d}}\right) \\
\vdots \\
u_{\mathrm{a}}^{(n-1)}\left(\left(1 / f_{\mathrm{d}}\right)^{-}\right)=u_{\mathrm{a}}^{(n-1)}\left(\left(1 / f_{\mathrm{d}}\right)^{+}\right) \\
u_{\mathrm{a}}^{(n-1)}\left(T_{\mathrm{p}}^{-}-1 / f_{\mathrm{d}}\right)=u_{\mathrm{a}}^{(n-1)}\left(T_{\mathrm{p}}^{+}-1 / f_{\mathrm{d}}\right)
\end{gathered}
$$

By introducing Equation (a.10) into (a.9), it yields:

$$
\begin{aligned}
& u_{\mathrm{a}}\left(0^{+}\right)=\dot{u}_{\mathrm{a}}\left(0^{+}\right) \ldots=u_{\mathrm{a}}^{(n-1)}\left(0^{+}\right)=0 \\
& u_{\mathrm{a}}\left(T_{\mathrm{p}}^{-}\right)=\dot{u}_{\mathrm{a}}\left(T_{\mathrm{p}}^{-}\right) \ldots=u_{\mathrm{a}}^{(n-1)}\left(T_{\mathrm{p}}^{-}\right)=0
\end{aligned}
$$

Conclusion $B: q^{\mathrm{i}}(t)$ is at least $C^{n}$ if $u_{\mathrm{a}}(t)$ is $C^{n-1}$ for its entire domain and $u_{\mathrm{a}}(t)$ and its $n-1$ first derivatives start and finish with a null value. 
b) $T_{\mathrm{p}}<1 / f_{\mathrm{d}}$

The different intervals of the analytical function are defined in Equation (a.12). Analogously, this function has been obtained with the time-domain convolution of Equation (17).

$$
q^{\mathrm{i}}(t)=\left\{\begin{array}{l}
0 \\
A_{\mathrm{d}} \int_{0}^{t} u_{\mathrm{a}}(\tau) \mathrm{d} \tau \\
A_{\mathrm{d}} \int_{0}^{T_{\mathrm{p}}} u_{\mathrm{a}}(\tau) \mathrm{d} \tau \\
A_{\mathrm{d}} \int_{t-1 / f_{\mathrm{d}}}^{T_{\mathrm{p}}} u_{\mathrm{a}}(\tau) \mathrm{d} \tau \\
0
\end{array}\right.
$$$$
\begin{aligned}
& t \leq 0 \\
& 0<t \leq T_{\mathrm{p}} \\
& T_{\mathrm{p}}<t \leq 1 / f_{\mathrm{d}} \\
& 1 / f_{\mathrm{d}}<t \leq T_{\mathrm{p}}+1 / f_{\mathrm{d}} \\
& T_{\mathrm{p}}+1 / f_{\mathrm{d}}<t
\end{aligned}
$$

Analogous to the previous case, we can verify that the same condition described in Conclusion $B$ applies when the duration $T_{\mathrm{p}}$ of $u_{\mathrm{a}}(t)$ is smaller than the oscillation period $1 / f_{\mathrm{d}}$.

\section{Multiplication by an exponential time function}

The motion profile $q^{\mathrm{i}}(t)$ is obtained either through Equation (17) or (18) when the pulse time-convolution technique is used. In both cases, it is required to include a product with a negative exponential time function $g(t)=\mathrm{e}^{-a t}$, which is a $C^{\infty}$ function. To study the effect of this operation with regard to the continuity degree, assume that $h(t)$ describes the product between $f(t)$ - an arbitrary function with continuity $C^{n}$ for all its domain - and $g(t)$ (Equation (a.13)). By following the same procedure described above, $h(t)$ is $n$-times differentiated (Equation (a.14)).

$$
\begin{gathered}
h(t)=f(t) g(t) \\
\dot{h}(t)=\dot{f}(t) g(t)+f(t) \dot{g}(t) \\
\vdots \\
h^{(n)}(t)=\sum_{k=0}^{n} \frac{n !}{k !(n-k) !}\left(f^{(n-k)}(t) g^{(n-k)}(t)\right)
\end{gathered}
$$

Notice that, in their domain, the sum of the functions is a continuous function if the addends are continuous. If one addend is discontinuous the sum is discontinuous. However, if two or more addends are discontinuous, it does not imply that the sum is necessarily a discontinuous function. Therefore, the continuity degree of a sum of functions is, at least the same as that of the one with lesser continuity. Moreover, the product of two functions is a continuous function if both are continuous and is discontinuous if one of them is discontinuous. However, if both terms are discontinuous one cannot conclude that the result is a discontinuous function. Therefore, the continuity degree of a product of two functions is again, at least the same as that of the one with lesser continuity.

According to (a.14), the $n$-times differentiated function $h^{(n)}(t)$ is a sum of terms that each one include a product of two functions $f^{(n-k)}(t) g^{(n-k)}(t), k=0,1 \ldots, n$. The continuity of each product is the same as the continuity of $f^{(n-k)}(t)$, because $g(t)$ is $C^{\infty}$. Moreover, the continuity degree of the sum $h^{(n)}(t)$ is at least the one from the most 
differentiated term, which is $f^{(n)}(t) g^{(n)}(t)$, for $k=0$. Therefore, the continuity of $h(t)$ is at least the same as $f(t)$.

\section{References}

[1] W.E. Singhose, Command Shaping for Flexible Systems: A Review of the First 50 Years. Int. J. Precis. Eng. Manuf., 10(4) (2009) 153-168. https://doi.org/10.1007/s12541-009-0084-2.

[2] M. Wu, J. Mei, Y. Zhao, W. Niu, Vibration reduction of delta robot based on trajectory planning. Mech. Mach. Theory, $153 \quad$ (2020), 104004. https://doi.org/10.1016/j.mechmachtheory.2020.104004.

[3] H. Ghorbani, B. Tarvirdizadeh, K. Alipour, A. Hadi, Near-time-optimal motion control for flexible-link systems using absolute nodal coordinates formulation. Mech. Mach. Theory, 140 (2019) 686-710. https://doi.org/10.1016/j.mechmachtheory.2019.06.032.

[4] O.J.M. Smith, Posicast Control of Damped Oscillatory Systems. Proceedings of the IRE, 45(9) (1957) 1249-1255. https://doi.org/10.1109/JRPROC.1957.278530.

[5] N.C. Singer, W.P. Seering, Preshaping Command Inputs to Reduce System Vibration. J. Dyn. Sys., Meas., Control, 112(1) (1990) 76-82. https://doi.org/10.1115/1.2894142.

[6] J.M. Hyde, W.P. Seering, Using Input Command Pre-Shaping to Suppress Multiple Mode Vibration. IEEE Proceedings of the International Conference on Robotics an Automation, Sacramento, CA, USA, 3 (1991) 2604-2609. https://doi.org/10.1109/ROBOT.1991.132020.

[7] W.E. Singhose, W.P. Seering, N.C. Singer, Shaping Inputs to Reduce Vibration: a Vector Diagram Approach. IEEE Proceedings of the International Conference on Robotics and Automation, Cincinnati, OH, USA, 2 (1990) 922-927. https://doi.org/10.1109/ROBOT.1990.126108.

[8] W.E. Singhose, W.P. Seering, N.C. Singer, Design and Implementation of Time-Optimal Negative Input Shapers. ASME Winter Annual Meeting, Chicago, IL, USA (1994) 151-157.

[9] N.C. Singer, W.P. Seering, An Extension of Command Shaping Methods for Controlling Residual Vibration Using Frequency Sampling. Proc. IEEE International Conference on Robotics and Automation, (1992) 800-805. https://doi.org/10.1109/ROBOT.1992.220271.

[10] W.E. Singhose, W.P. Seering, N.C. Singer, Input Shaping for Vibration Reduction with Specified Insensitivity to Modeling Errors. Proceedings of the 1996 JapanUSA Symposium on Flexible Automation, (1996) 307-313.

[11] P.H. Meckl, W.P. Seering, Minimizing Residual Vibration for Point-to-Point Motion. J. Vib., Acoust., Stress, and Reliab., 107(1) (1985) 378-382. https://doi.org/10.1115/1.3269276.

[12] P.H. Meckl, W.P. Seering, Reducing Residual Vibration in Systems with Time-Varying Resonances. IEEE International Conference on Robotics and Automation, Raleigh, NC, USA, $4 \quad$ (1987) 1690-1695. https://doi.org/10.1109/ROBOT.1987.1087879.

[13] P.H. Meckl, P.B. Arestides, M.C. Woods, Optimized S-Curves Motion Profiles for Minimum Residual Vibration. IEEE Proceedings of the American Control Conference, Philadelphia, PA, USA, 5 (1998) 2627-2631. https://doi.org/10.1109/ACC.1998.688324. 
[14] W.E. Singhose, N.C. Singer, W.P. Seering, Comparison of Command Shaping Methods for Reducing Residual Vibration. Proceedings of the European Control Conference, Rome, Italy (1995) 1126-1131.

[15] S.P. Bhat, D.K. Miu, Precise Point-to-Point Positioning Control of Flexible Structures. J. Dyn. Sys., Meas., Control, 112(4) (1990) 667-674. https://doi.org/10.1115/1.2896193.

[16] S.P. Bhat, M. Tanaka, D.K. Miu, Experiments on Point-to-Point Position Control of a flexible Beam Using Laplace Transform Technique-Part 1: Open-Loop. J. Dyn. Sys., Meas., Control, $113 \quad$ (1991) 432-437. https://doi.org/10.1115/1.2896428.

[17] T.D. Tuttle, W.P. Seering, A Zero-Placement Technique for Designing Shaped Inputs to Suppress Multiple-Mode Vibration. Proceedings of the American Control Conference, Baltimore, MD, USA (1994) 2533-2537. https://doi.org/10.1109/ACC.1994.735015.

[18] J.M. Veciana, S. Cardona, Residual vibration reduction in mechanical systems: A time-domain approach. Int. J. Precis. Eng. Manuf., 13(8) (2012) 1327-1339. https://doi.org/10.1007/s12541-012-0176-2.

[19] B.R. Murphy, I. Watanabe, Digital Shaping Filters for Reducing Machine Vibration. IEEE Transactions on Robotics and Automation, 8(2) (1992) 285-289. https://doi.org/10.1109/70.134281.

[20] W. E. Singhose, J. Vaughan, Reducing Vibration by Digital Filtering and Input Shaping. IEEE Transactions on Control Systems Technology, 19(6) (2011) 14101420. https://doi.org/10.1109/TCST.2010.2093135.

[21] G. Farin, Curves and Surfaces for Computer Aided Geometric Design. A Practical Guide. Academic Press, Inc, 1997.

[22] E. Lores, Aportació a l'estudi de la reducció de vibracions residuals en transitoris de moviment generats mitjançant accionaments de cinemàtica no lineal. $\mathrm{PhD}$ thesis, UPC, Barcelona, 2017.

[23] E. Lores, J.M. Veciana, L. Jordi, Feasibility of motion laws for planar one degree of freedom linkage mechanisms at dead point configurations. Mech. Syst. Signal Process., 98 (2018) 834-851. https://doi.org/10.1016/j.ymssp.2017.05.015.

[24] J.R. Brauer, Magnetic Actuators and Sensors. John Wiley \& Sons, 2006. https://doi.org/10.1002/0471777714.

[25] S. Cardona, D. Clos, Teoria de Màquines. Edicions UPC, Barcelona, 2001.

[26] B. Paul, Kinematics and Dynamics of Planar Machinery. Prentice-Hall, Inc, 1979.

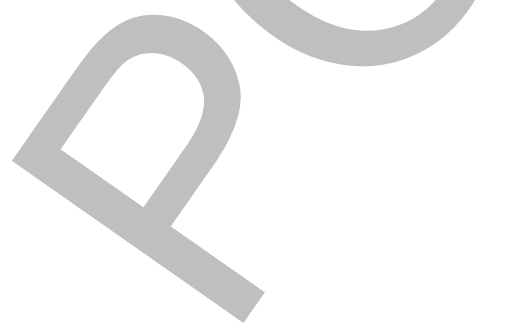

